\title{
Chapter 19 \\ Thermodynamics of Distinguishable Particles: A Key to High-Energy Strong Interactions?
}

\section{Rolf Hagedorn}

\begin{abstract}
A new kind of thermodynamical model for strong interactions at high energies is proposed. We start from the fact that strong interactions produce so many possible particle states (from $\pi$ over its resonances to nucleons, strange particles and their resonances, up to highly excited 'fireballs') that in an actual process each of these states practically never occurs more than once. We use this in order to treat the very first instant of a high-energy collision by statistical thermodynamics of a system of an illimited number of distinguishable particles. The model shows surprising properties: there exists a universal highest possible temperature $T_{0}$ of the order of $150-200 \mathrm{MeV}$ (corresponding to $\approx 10^{12} \mathrm{~K}$ ) which governs all highenergy processes of strongly interacting particles, independently of the actual energy and independently of the particle number, from cosmic ray jets down to elastic scattering. If a Lorentz contracted volume is introduced, the transverse momentum distribution in jets as well as in elastic scattering is described in agreement with experimental results. Paradoxically, this distribution is independent of whether or not 'thermal equilibrium' is reached. If it is not reached-in the majority of cases it is not reached-then the jet structure for production processes is the consequence. If the model turns out to be as good as present experiments indicated, then the existence of a highest temperature is very likely; it implies that, from higher and higher energy experiments, not much new can be learnt about the structure of strong interactions, since the mode of excitation (which depends on the dynamical details we would like to know) has no influence on what is finally observed. The situation would then be similar to that in ordinary thermodynamics, where no experiment could possibly reveal how a certain system was brought into its thermodynamical state. In astrophysics, the method of thermodynamics of distinguishable particles may have important consequences for the treatment of the highly compressed
\end{abstract}

Preprint CERN-TH-483, October 1964,

http://cds.cern.ch/record/929730?1n=en archived in electronic format in year 2006/2010.

R. Hagedorn: (deceased) CERN-TH, 1211 Geneve 23, Switzerland

J. Rafelski $(\bowtie)$

Department of Physics, The University of Arizona, Tucson, AZ 85721, USA 
interior of heavy stars ('neutron stars') where Fermi statistics would have to be replaced by the one used here.

\subsection{Introduction}

In the past 10-15 years, it has become a more and more established (and more and more accepted) habit to publish conjectures, models, speculations-no matter whether:

- the foundations are safe,

- or all features have been worked out and compared with experiments,

- or even the correct physical interpretation of the resulting formulas is understood.

The model presented here suffers from all these deficiencies - and so far it is in good company. This is the first excuse to publish it. The second is that it shows some very remarkable features agreeing with some experimental facts and that there is a hope that it can be brought into a state where it becomes a theory. Whether it will survive this development is an open question. The few striking features to be presented below seem to make it interesting enough to publish it and initiate a discussion.

There are two roots of the present model:

1. Root one is a well-known observation already made by Fermi [1] in his first paper on the statistical model: the statistical model of particle production starts from expressions for channel probabilities (final channel $f_{j}$ with $N_{j}$ particles):

$$
\begin{aligned}
P_{j} & =\int\left|\left\langle f_{j}|T| i\right\rangle\right|^{2} \prod_{k=1}^{N_{j}} \delta\left(p_{k}^{2}-m_{k}^{2}\right) \mathrm{d}^{4} p_{k} \\
& =\left(\prod_{k=1}^{N_{j}} C_{k}\right) F_{\text {stat }} \int \delta^{4}\left(p_{i}-\sum_{i}^{N_{j}} p_{k}\right) \prod_{1}^{N_{j}} \frac{\mathrm{d}^{3} p_{k}}{2 E_{k}} \\
& =\left(\prod_{k=1}^{N_{j}} \Omega_{k}\right) F_{\text {stat }} \int \delta^{4}\left(p_{i}-\sum_{i}^{N_{j}} p_{k}\right) \prod_{1}^{N_{j}} \mathrm{~d}^{3} p_{k},
\end{aligned}
$$

where $\prod_{k=1}^{N_{j}} C_{k}$ and $\prod_{k=1}^{N_{j}} \Omega_{k}$ stand for the mean values of the squared matrix element with respect to the invariant or non-invariant phase space, respectively, and $F_{\text {stat }}$ takes into account spin and isospin weight and contains $1 / n_{i}$ ! when $n_{i}$ particles of type $i$ are present. It was then observed by Fermi that a statistical model starting from Eq. (19.1) fits smoothly into a thermodynamical model once the energy $E$ and thus the particle number become large enough. Thus, in discussing high-energy limits, one will use conveniently the methods of statistical thermodynamics which are far easier to handle than the expressions in Eq. (19.1). 
2. The second root of the present model is the observation that a very particular kind of statistical thermodynamics is necessary to fit the actual behaviour of the statistical model of type (19.1): it was found by evaluating numerical calculations made at CERN (1958-1962) at various c.m. energies (using the non-invariant phase space) that the ratio of the probability $P_{0}$ for the elastic channel to the sum over all probabilities behaves like an exponential function of the c.m. energy over a fairly large range [2] $(3 \leq E \leq 7.6)$ :

$$
\left.\frac{p_{0}}{\sum p_{j}}\right|_{\mathrm{pp}}=\mathrm{e}^{-3.10(E-2)}
$$

with units such that $\hbar=c=k=M_{p}=1, k$ is Boltzmann's constant. This, and a similar result for $\pi \mathrm{p}$ collisions, was used to predict large angle elastic $[2,3]$ and exchange scattering, i.e., $p+p \rightarrow \mathrm{A}+\mathrm{B}$, etc. [4]. The predictions for $p p$ elastic scattering at about $90^{\circ}$ fit the experiments qualitatively over a range where the cross-section changes by 5 powers of ten.

It was natural to ask the question whether the exponential behaviour could be understood analytically since Eq. (19.2), being the result of hundreds of hours of computer time, is practically an empirical result. This question was attacked by several authors [5-8]. Bialas and Weisskopf [6] obtained ${ }^{1}$

$$
\frac{p_{0}}{\sum p_{j}} \sim \mathrm{e}^{-\alpha(E-2)^{3 / 4}}, \quad \alpha \text { a constant },
$$

starting directly from statistical thermodynamics. Satz [8] considered the asymptotic behaviour for $E \rightarrow \infty$ of $\sum p_{j}$ with $p_{j}$ in the form of Eq. (19.1) and found

$$
\frac{p_{0}}{\sum p_{j}} \sim \mathrm{e}^{-a E^{2 / 3}}
$$

Vandermeulen [7] considered a special case of Eq. (19.1), namely the one where all masses are neglected (which can be justified), and found

$$
\frac{p_{0}}{\sum p_{j}} \sim \mathrm{e}^{-b E}
$$

All the authors mentioned so far started from definite assumptions and obtained definite results which do not all agree with Eq. (19.2), although in one case it could be shown that, in the limited range where Eq. (19.2) was computed, expressions Eqs. (19.2) and (19.3) deviate little numerically from each other (see Fig. 4 of Bialas and Weisskopf [6]).

\footnotetext{
${ }^{1}$ Here and in the following quotations, we consistently neglect algebraic expressions in $E$ as compared to the exponential.
} 
For our present discussion, the most interesting paper is that by Auberson and Escoubès [5], because these authors discuss various different assumptions and find different forms, namely,

$$
\frac{p_{0}}{\sum p_{j}} \sim \mathrm{e}^{-c E^{\alpha}}, \quad \alpha=\frac{1}{2}, \frac{2}{3}, \frac{3}{4}, 1,
$$

according to what the assumptions are. In order to be close to the calculations leading to Eq. (19.2), they work with the non-invariant phase space. An overall result is, whatever the particular assumptions are, that the masses of the particles produced can be neglected when $E \rightarrow \infty$. (Except if the main contributions come from ever new 'particles' with higher and higher mass values as $E$ increases, such that $E \bar{m}$ remains constant, $\bar{m}$ being the mean value of the masses produced. This is not at all likely in view of the purely geometrical fact that the phase space for small or negligible masses is so much bigger than for masses such that $E \bar{m}$ is constant; only some very peculiar dynamical properties, which so far we have no reason to expect, would be able to counteract this tendency of phase space.) A particular result is that

$$
\sum p_{j}=\sum_{n=2}^{\infty}\left[\frac{\Omega^{n}}{n !} \int \delta\left(E-\sum_{i=1}^{n} \varepsilon_{i}\right) \delta^{3}\left(\sum_{i=1}^{n} \mathbf{p}_{i}\right) \mathrm{d}^{3} p_{1} \ldots \mathrm{d}^{3} p_{n}\right]
$$

tends asymptotically to $\mathrm{e}^{c E}$ only if the factor of $1 / n$ ! is omitted, in other words, if the particles are considered to be distinguishable. ${ }^{2}$

This is not as unreasonable as it first sounds. And since it is the main point of the model which will be presented below, we must explain this more carefully. Auberson and Escoubès seek an asymptotic formula for the statistical model which fits the detailed numerical calculations at moderately high energies. In these detailed calculations, many different particles were considered $\left(\Xi^{-}, \Xi^{0}, \Sigma^{+}, \Sigma^{0}, \Sigma^{-}, \Lambda, \mathrm{p}, \mathrm{N}\right.$, $\mathrm{N}_{3 / 2}^{*}, \mathrm{~K}^{0}, \mathrm{~K}^{+}, \mathrm{K}^{-}, \pi^{+}, \pi^{0}, \pi^{-}$, and in some calculations $\rho, \omega$, and $\eta$ ). It turned out that the calculated average particle numbers hardly exceeded the value one, and even for the pions they remained below two (per charge state). Had we included all the presently known resonances, then all average occupation numbers of the various states of the particles would have remained below one. Now in those calculations the factor $1 / n$ ! actually takes the form $1 / n_{1} ! n_{2} ! \ldots$, where $n_{1}, n_{2}$, etc., are the numbers of particles of type 1 , type 2 , etc. $^{3}$ Since then $\left\langle n_{i}\right\rangle$ actually turns out to be $\lesssim 1$, it follows that mainly those channels contribute to $\sum p_{j}$, where the $n_{i}$ are either 0 or 1 , in other words, where the whole factor $1 / n_{1} ! n_{2} ! \ldots$ equals one.

\footnotetext{
${ }^{2}$ In fact, $\Omega$ must also be kept independent of $E$, contrary to what was done in the numerical calculations leading to Eq. (19.2). But there the masses were not neglected, and one sees easily that at moderate energies this will have the effect of increasing the power of $E$ in the exponential (the details are difficult).

${ }^{3}$ Here, $\pi^{+}, \pi^{0}$, and $\pi^{-}$, etc., are of course considered to be different particles.
} 
Since we presently know many more states of the fundamental particles, and since it seems likely that higher and higher excited states may be found, we expect that feeding them into the statistical model will have the effect that, even at very high energies, the main contributions will come from channels in which the $n_{i}$ are zero or one. If therefore a simplified model of the type given in Eq. (19.7) with particles of equal masses is used to find out the asymptotic behaviour, then one should omit the factor $1 / n$ ! in order to come as near as possible to reality. It is reassuring that just in doing that one finds the exponential behaviour which is indicated by our numerical result (19.2) and which fits the experiment [9].

As one should expect, statistical thermodynamics of massless particles-all distinguishable from each other!-leads then to the same behaviour. The main formulas for such a statistics were worked out by Escoubès and the present author and included in the paper by Auberson and Escoubès [5].

In the following, we shall therefore discuss the model 'statistical thermodynamics of distinguishable particles' in some detail and try to understand its physical meaning. It should be clear from our considerations that the mechanism, which we imagine to take place, is the following. In the first instant of the collision, a certain number of particles - ranging from pions over kaons, nucleons, hyperons and their resonances to highly excited 'fireballs'-is produced according to the statistics of distinguishable particles. Then resonances decay according to their mode and 'fireballs' decay again according to the statistics of distinguishable particles, each one forming such a system. At the end of this chain of decays we arrive at pions, kaons, nucleons, and hyperons, where now the number of pions may be much larger than one without invalidating our treating the particles as distinguishable.

In Sect. 19.2, we present the simplest possible model of this kind. In Sect. 19.3, we speculate on its physical interpretation which, sometimes, is rather obscure. In Sect. 19.4, we discuss its weak points and possible improvements, and in Sect. 19.5, we sum up and draw a few general conclusions.

\subsection{Statistical Thermodynamics of Distinguishable Particles}

We write down the assumptions:

1. We consider a system of particles enclosed in a volume $V$ in a temperature bath $T$. [We put the Boltzmann constant $k=1$, the temperature $T$ is then measured in nucleon masses: $T=1(=939 \mathrm{MeV})$ in those units corresponds to $1.1 \times 10^{13} \mathrm{~K}$.]

2. The number of particles is not limited.

3. All particles are distinguishable, i.e., can be labelled.

4. All particles have mass zero (we shall later consider massive particles) and no internal (mechanical) degrees of freedom (the $2 s+1$ possible orientations of a particle with spin $s$ are considered as $2 s+1$ different particles).

Let $\varepsilon_{1}, \varepsilon_{2}, \ldots, \varepsilon_{\alpha}, \ldots$, be the possible energy levels of one particle in the volume $V$. If we give a set of numbers $(n)=\left(n_{1}, n_{2}, \ldots, n_{\alpha}, \ldots\right)$ indicating by $n_{\alpha}$ how many particles of energy $\varepsilon_{\alpha}$ are present, then, in the usual case of indistinguishable 
particles, $(n)$ would completely specify a quantum state of our gas. But as we consider the particles to be distinguishable, $(n)$ stands for

$$
\frac{N !}{n_{1} ! n_{2} ! \ldots n_{\alpha} ! \ldots}
$$

different states of the same energy $E=\sum_{\alpha} n_{\alpha} \varepsilon_{\alpha}$, with $N=\sum_{\alpha} n_{\alpha}$. For $N$ particles, the partition function will then be

$$
\begin{aligned}
Z_{N} & =\sum_{(n)} \frac{N !}{n_{1} ! n_{2} ! \ldots} \exp \left(-\frac{1}{T} \sum n_{\alpha} \varepsilon_{\alpha}\right) \quad\left(N=\sum n_{\alpha}\right) \\
& =\left(\sum_{\alpha} \mathrm{e}^{-\varepsilon_{\alpha} / T}\right)^{N} \quad\left(\text { with } N=\sum_{\alpha} n_{\alpha}\right. \text { fixed). }
\end{aligned}
$$

We shall use the shorthand notations

$$
x_{\alpha} \equiv \mathrm{e}^{-\varepsilon_{\alpha} / T}, \quad z \equiv \sum_{\alpha} \mathrm{e}^{-\varepsilon_{\alpha} / T}=\sum_{\alpha} x_{\alpha} .
$$

We calculate $z$ for a massless particle $(p=\varepsilon)$ in the usual way:

$$
g(p) \mathrm{d} p=g(\varepsilon) \mathrm{d} \varepsilon=\frac{4 \pi p^{2} \mathrm{~d} p V}{h^{3}}
$$

as density of states gives in our units

$$
z=\int g(\varepsilon) \mathrm{e}^{-\varepsilon / T} \mathrm{~d} \varepsilon=\frac{V T^{3}}{\pi^{2}} .
$$

We now drop the assumption that $N$ is fixed and find

$$
Z=\sum_{N=0}^{\infty} Z_{N}=\sum_{N=0}^{\infty} z^{N}=\frac{1}{1-z}=\frac{1}{1-\sum x_{\alpha}}=\frac{1}{1-V T^{3} / \pi^{2}}
$$

for the partition function of our system. We observe here the striking feature, which will be of fundamental importance and indeed the very heart of our model, namely, that the partition function of our gas exists only if the temperature

$$
T<T_{0}=\left(\frac{\pi^{2}}{V}\right)^{1 / 3}
$$

We now calculate the expectation values of the energy $E$ and particle number $N$ of our system (as the system is in thermal contact with a temperature bath $T$, its energy 
is not fixed: our system is a member of a canonical, not microcanonical ensemble). We find from Eq. (19.11) that

$$
\bar{E}=T^{2} \frac{\partial}{\partial T} \log Z=\frac{3 V T^{4}}{\pi^{2}} \frac{1}{1-V T^{3} / \pi^{2}}=Z \frac{3 V T^{4}}{\pi^{2}} .
$$

(Note that ordinary Bose statistics would give

$$
\frac{1}{2}\left(\frac{V \pi^{2}}{15} T^{4}\right)
$$

per internal degree of freedom, i.e., the Stefan-Boltzmann law. Our gas would, for $T \rightarrow 0$ thus behave like a light quantum gas with a slightly changed radiation constant.) We thus see that $\bar{E}$ diverges when $T \rightarrow T_{0}$.

The average occupation number of the energy level $\varepsilon_{\alpha}$ becomes with Eq. (19.11)

$$
\bar{n}_{\alpha}=x_{\alpha} \frac{\partial}{\partial x_{\alpha}} \log Z=\frac{\mathrm{e}^{-\varepsilon_{\alpha} / T}}{1-V T^{3} / \pi^{2}}=Z \mathrm{e}^{-\varepsilon_{\alpha} / T} .
$$

This also determines the energy (= momentum) spectrum (see below). The expectation value of the particle number is

$$
\bar{N}=\sum_{\alpha} \bar{n}_{\alpha}=z Z=\frac{V T^{3}}{\pi^{2}\left(1-V T^{3} / \pi^{2}\right)}=Z \frac{V T^{3}}{\pi^{2}}
$$

and the average energy (= momentum) of a particle is

$$
\bar{\varepsilon}=\frac{\bar{E}}{\bar{N}}=3 T
$$

We observe that, in Eqs. (19.13)-(19.15), the relevant quantities all contain $Z$ and therefore diverge when $T \rightarrow T_{0}$. Since we will be concerned throughout this paper with large energies, we consider the behaviour near $T=T_{0}$. Then in the slowly varying factors, $T$ may be replaced by $T_{0}$ and we obtain the simple expressions

$$
\bar{E} \rightarrow 3 T_{0} \frac{T_{0}^{3}}{T_{0}^{3}-T^{3}}, \quad \bar{N} \rightarrow Z=\frac{T_{0}^{3}}{T_{0}^{3}-T^{3}}, \quad \bar{n}_{\alpha} \rightarrow \mathrm{e}^{-\varepsilon_{\alpha} / T_{0}} \frac{T_{0}^{3}}{T_{0}^{3}-T^{3}}
$$

Let us draw $\bar{E}$ (or $Z$ ) as a function of $T$, or better, $T$ as a function of $\bar{E}$. We obtain the behaviour shown in Fig. 19.1, where we omit the unphysical temperatures $T>T_{0}$ which lead to negative $\bar{E}$. This figure has obviously to be intepreted as follows: as soon as $T$ comes very near $T_{0}$, we may achieve any (large) values of $\bar{E}, \bar{N}, Z$ by only infinitesimal changes in the temperature. Consequently, as the mean values $\bar{E}$ and $\bar{N}$ suffer enormous changes for infinitesimal changes in $T$, we no longer expect the 


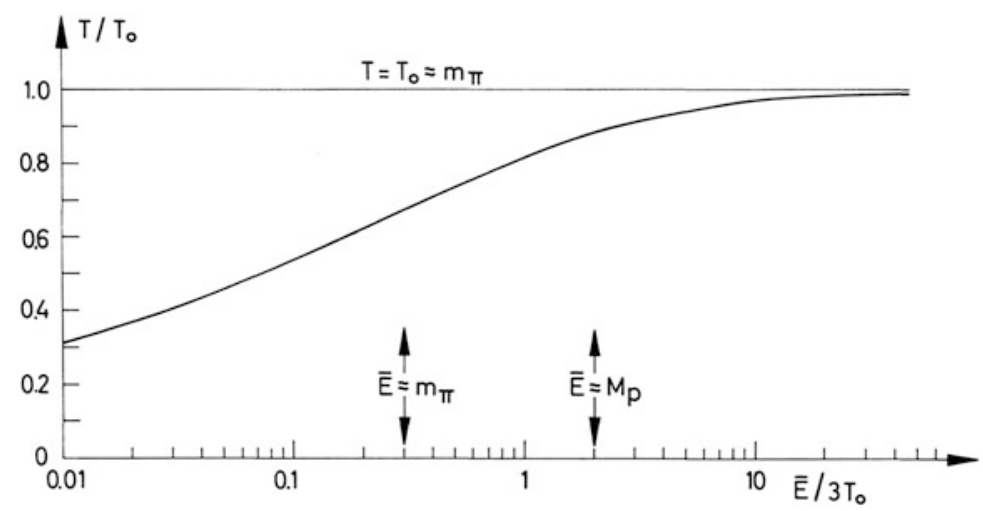

Fig. 19.1 Temperature $T / T_{0}$ as a function of the energy $\bar{E} / 3 T_{0}$ for particles of zero mass

distributions of $E$ and $N$ over our canonical ensemble to have the sharp peaks we are used to in statistical thermodynamics. On the contrary, these distributions become flat and tend to a constant when $T \rightarrow T_{0}$. Indeed, as follows from the definition of $Z$ [see Eqs. (19.8) and (19.11)], we have

$$
T^{2} \frac{\mathrm{d} \bar{E}}{\mathrm{~d} T}=\overline{E^{2}}-\bar{E}^{2}, \quad z \frac{\mathrm{d} \bar{N}}{\mathrm{~d} z}=\overline{N^{2}}-\bar{N}^{2},
$$

which gives, when evaluated for $T \rightarrow T_{0}$ and $z \rightarrow 1$,

$$
\frac{\overline{E^{2}}-\bar{E}^{2}}{\bar{E}^{2}} \longrightarrow 1, \quad \frac{\overline{N^{2}}-\bar{N}^{2}}{\bar{N}^{2}} \longrightarrow 1 .
$$

It is easily seen that the distribution of $N$ becomes a constant. Writing

$$
\bar{N}=\frac{\sum N z^{N}}{\sum z^{N}}
$$

gives at once the probability of finding $N$ particles as

$$
W(N)=\frac{z^{N}}{\sum z^{N}}=\frac{z^{N}}{Z} .
$$

Since $z \rightarrow 1$ and $Z \rightarrow \infty$ when $T \rightarrow T_{0}$, we obtain $W(N) \rightarrow 0$. In other words, when $T \rightarrow T_{0}$, the energy and particle number of our system become 
undetermined. ${ }^{4}$ Thus, from the point of view we have adopted so far, namely to consider our system as a member of a canonical ensemble (or as being in a temperature bath), we have failed to achieve anything and would be obliged to stop here. We shall see, however, in the next section, that just those circumstances which make it impossible to follow further the lines of the usual interpretation will serve to allow a new interpretation, if only by brute force.

\subsection{The Interpretation of the Model}

If, in statistical thermodynamics, we encountered a case where the mean energy of a system in a temperature bath $T$ were undetermined, we would say that the system considered were not suited for a statistical treatment. A similarly unfavourable situation would, for instance, arise if a system contained, say, only two atoms: their total energy would be rather badly determined —or, if we fixed its energy in an ad hoc manner and considered it as a member of a microcanonical ensemble, we could hardly speak of its temperature.

In our present model, however, the situation is different: the very fact that for $T \rightarrow T_{0}$ the system can have any energy and that $\bar{E}$ becomes very large can be reinterpreted as follows. Whenever a system is given-whose energy $E$ is fixed and sufficiently large, namely $E \gg T_{0}$-then we may think of it as a former member of (and now isolated from) the canonical ensemble embedded in a temperature bath $T \rightarrow T_{0}$. We may then forget about the canonical ensemble and simply postulate that we can ascribe to any system of sufficiently large energy the temperature $T_{0}$, even if the system chooses to have a small number of particles. By inverting Eq. (19.17), we have $T$ as a function of $\bar{E}$ :

$$
T=T_{0}\left(1-\frac{T_{0}}{\bar{E}}+\cdots\right),
$$

so that $T \approx T_{0}$ whenever $\bar{E} \gg T_{0}$. Although this holds for the dependence of $T$ on $\bar{E}$ and although we have seen that for $T \rightarrow T_{0}$ we might expect any, even small, energies, we play safe when we say that we ascribe to the system the temperature $T_{0}$ if it has an actual energy $E \gg T_{0}$; if the energy is small, it still could have belonged to the ensemble of temperature $T_{0}$, but it could as well have belonged to a lower temperature.

Next we observe that only $\bar{E}$ and $\bar{N}$ are (via $T$ ) coupled to each other, but not the actual energy $E$ and particle number $N$ of a system. Thus, once we have fixed $E \gg T_{0}$ and ascribed the temperature $T_{0}$ to the system, we may still expect any number $N$ of particles; indeed, all $N$ values become equally likely for $T \rightarrow T_{0}$.

\footnotetext{
${ }^{4}$ The relative fluctuations of the occupation probabilities $\bar{W}_{\alpha}=\overline{\left(n_{\alpha} / N\right)}$ vanish, however. Hence, $\bar{W}_{\alpha}$ becomes 'sharp' when $T \rightarrow T_{0}$ (see Appendix 1).
} 
By the reinterpretation of our model, we have of course abandoned the point of view of the canonical ensemble of temperature $T$ and have obtained the description of a single system of given energy $E$. This system must no longer be thought of as being in contact with its surroundings - which would be hard to imagine for a highenergy collision - but, thanks to the peculiar behaviour of $\bar{E}(T)$, it has a temperature $T \rightarrow T_{0}$ in its own right.

As for the value of $T_{0}=\left(\pi^{2} / V\right)^{1 / 3}$, it must be chosen such that, at least, the system itself conserves the main features of a thermodynamical system: the particles must be able to interact with each other. As we wish to describe highenergy collisions of strongly interacting particles, where the particles produced will escape radially from the region of interaction, they will cease to interact once their mutual distances become much larger than the range of forces, i.e., the Compton wavelength of the pion. Thus the volume $V$ is to be taken as

$$
V=\frac{4 \pi}{3}\left(\frac{a}{m_{\pi}}\right)^{3},
$$

where $a \approx 1$ is an adjustable parameter. With Eq. (19.12), we obtain

$$
T_{0}=\frac{m_{\pi}}{a}\left(\frac{3 \pi}{4}\right)^{1 / 3}=m_{\pi} \frac{1.35}{a} \equiv b m_{\pi},
$$

where $b=1.35 / a$ is again of order one.

We now formulate the new interpretation in the following postulate.

Postulate To every high-energy collision of strongly interacting particles (hadrons) of total centreof-mass (kinetic) energy $E \gg m_{\pi}$, and also to every highly excited hadron ('fireball') with excitation $\Delta E \gg m_{\pi}$, we ascribe the temperature $T \rightarrow T_{0}=b m_{\pi}$ with $b \approx 1$.

We can consider this temperature $T \approx 140 \mathrm{MeV}$ (corresponding roughly to $10^{12} \mathrm{~K}$ ) as the 'highest possible temperature' which, as a fundamental constant, governs all high-energy processes of strongly interacting particles. [Of course, this goes as far as the present simplified model is valid-introduction of masses and of a particular shape of the volume of interaction and other refinements may change this conclusion somewhat (see below).] Apart from such, we hope, minor changes, we would predict on this basis that $T_{0}$ will come into play whenever at least one strongly interacting particle takes part in a collision. Therefore high-energy reactions with initial states like $\mathrm{e}+p, \gamma+p, \nu+p$, and those in which $\mathrm{p}$ is replaced by any other hadron, will show certain features similar to $p+p$ collisions at high energies. The reason is that, whenever a sufficient amount of excitation energy $\left(\Delta E \gg T_{0}\right)$ is transferred to a hadron, the excited hadron falls under the above postulate. This holds even for such reactions as $\mathrm{e}+\mathrm{e} \rightarrow \mathrm{e}+\mathrm{e}+$ hadrons. For two reasons, this does not apply to the weakly or electromagnetically interacting partners of the reaction:

- They have no reason to feel the temperature $T_{0}$ which has its origin in the range of strong interactions. 
- They do not interact strongly enough to produce the many resonances required for a statistics of distinguishable particles. For indistinguishable particles, no $T_{0}$ exists.

It is interesting to compare these immediate consequences of our model (and of the above postulate) with recent speculations by Wu and Yang [10]. They assume that the sharp decrease with energy of differential cross-sections at large angles is due to a mechanism independent of the method of excitation and discuss the consequences of such a possibility. Our present model provides a natural basis for their assumption and leads to the same consequences (which were also partly drawn by the present author [4]).

We shall now draw some quantitative conclusions of our postulate and try to see how our system will behave experimentally. First we remark that, since we now consider the energy $E$ to be given, and work in the centre-of-mass frame, we have to impose energy-momentum conservation, at least when the number of particles is small. In particular, we should extend the summation $Z=\sum z^{N}$ from $N=1$ to $\infty$ and not-as we did above-from $N=0$ to $\infty$. We shall keep this point in mind. It does, however, not change our conclusions about the gross features of the system. Since we work at $T=T_{0}$, the partition function diverges and it does not matter, in general, whether or not the first term is included.

Secondly, we remark that, putting all masses equal to zero is an oversimplification in some cases. For instance, the total energy $E$ has then often to be interpreted as kinetic energy. Our assumption that the particles be distinguishable is based on the experimental fact that so many particle states (resonances, charge, hypercharge) are known and that the list of them grows steadily. Of course, the newly added particles have a tendency to have higher and higher mass values - it may well be that the 'fireballs' of cosmic ray events are the asymptotic form of them where the widths of the resonances are larger than their spacing. We shall consider here everything from a pion over kaon, nucleon, hyperon, and resonances up to the fireballs, as possible 'particles' appearing in our system, and this would force us to take the mass of such a particle into account. But presently, we shall simply put $m=0$ and $T=T_{0}$ (if necessary, $\lim _{T \rightarrow T_{0}}$ is understood).

We shall consider:

- the number of particles produced (first generation), ${ }^{5}$

- the momentum spectrum of the particles (first generation).

These two are related to each other and treated together. From Eq. (19.14), we have for the expectation value of the number of particles with energy $\varepsilon_{\alpha}$,

$$
\bar{n}_{\alpha}=Z \mathrm{e}^{-\varepsilon_{\alpha} / T_{0}}
$$

\footnotetext{
5 'First generation' means the distinguishable particles (produced in the first instant) which later, by a chain of further 'generations', decay into the observed pions, nucleons, etc.
} 
and from this

$$
\bar{N}=\sum \bar{n}_{\alpha}
$$

Now, however, we must insist on energy conservation, that is, these formulas are subject to the condition

$$
\sum \varepsilon_{\alpha} \bar{n}_{\alpha}=E
$$

The usual definition of $\bar{E}$, which we used in Eq. (19.13), namely

$$
\bar{E}=T^{2} \frac{\partial}{\partial T} \log Z
$$

can be derived from the requirement $\sum \varepsilon_{\alpha} \bar{n}_{\alpha}=\bar{E}$ with $\bar{n}_{\alpha}$ defined by Eq. (19.14). We are thus forced, if we insist on energy conservation which leads to Eq. (19.25), to identify the expectation value $\bar{E}$ of the old interpretation (canonical ensemble) with the given sharp value $E$ of the energy in our new interpretation. Consequently, wherever $Z$ and $\bar{E}$ appear explicitly, we shall replace them by

$$
\bar{E} \longrightarrow E, \quad Z \longrightarrow E \frac{\pi^{2}}{3 V T_{0}^{4}}=\frac{E}{3 T_{0}},
$$

as suggested by Eqs. (19.10), (19.12), (19.13), and (19.25). Then with (19.21), the temperature $T$ becomes

$$
T=T_{0}\left(1-\frac{T_{0}}{E}+\cdots\right) \approx T_{0},
$$

and the number of particles with energy $\varepsilon_{\alpha}$ is

$$
\bar{n}_{\alpha}=\frac{E}{3 T_{0}} \mathrm{e}^{-\varepsilon_{\alpha} / T_{0}}, \quad \bar{N}=\sum \bar{n}_{\alpha}=\frac{E}{3 T_{0}} .
$$

Our conclusion that the distribution of $N$ values becomes constant remains true. We can even see how it tends to the constant $z=V T^{3} / \pi^{2}$ and $T$ from Eq. (19.27) gives $z(Z) \simeq 1-3 T_{0} / E\left(E \gg T_{0}\right)$. Hence, for the probability $W(N)$ of finding just $N$ particles, Eq. (19.20) yields

$$
W(N) \cong \frac{3 T_{0}}{E}\left(1-\frac{3 T_{0}}{E}\right)^{N} \approx \frac{3 T_{0}}{E} \exp \left(-N \frac{3 T_{0}}{E}\right) \equiv \frac{1}{N} \mathrm{e}^{-N / \bar{N}} \longrightarrow 0
$$

Now this seems to be in spectacular disagreement with experiments: neither-as we know from cosmic ray evidence-does the number $\bar{N}$ of particles produced 
increase proportionally to $E$, nor is the distribution of the observed particle number constant. The way out of this apparent disagreement is again provided by the model itself: the $N$ particles found in a particular case are by no means the final particles observed in photographic emulsions (mainly pions)—speaking of distinguishable particles, we have to consider them to be anything between a pion and a 'fireball'. Equation (19.29) gives us the probability of just finding $N$ such not further specified objects. It states that all values of $N$ are (for $N \sim \bar{N}$ ) practically equally likely. The question how probable is it to find $N$ specified particles is quite another one, as we shall see in a moment when we discuss large angle elastic scattering. Presently, we note that, interpreting the $N$ particles as 'fireballs' of unspecified excitation energy, Eq. (19.29) tells us that a 'two-fireball model' would never work, ${ }^{6}$ as there will be contributions of almost the same weight from $3,4,5, \ldots$, fireballs, a situation similar to that in the multiperipheral model of Amati et al. [12] and in considerations by Wilson [13].

But if this is so, then the number of pions and other final particles observed in experiments should even be larger than $\bar{N}=E / 3 T_{0}$, since these particles are produced in a chain of decays starting from the first $N$ 'fireballs' and going into smaller and smaller ones. Here the answer is that introducing the masses and a contracted volume will bring that in order: we shall come back to this problem in Sect. 19.4, where it will be shown that $\bar{N}$, the number of 'fireballs', tends to $\approx 5$ and becomes energy independent for $E \rightarrow \infty$.

Let us now consider the energy spectrum of our particles. First we treat the case where the question of how many particles we expect and the question of what their energies might be are intimately connected: large angle elastic and exchange scattering. In that case, we have two definite final particles, each with energy $E / 2$, and from Eq. (19.28), we conclude that the probability of finding a particle in the energy level $\varepsilon_{\alpha}=E / 2$ is given by

$$
w_{\alpha}=\frac{\bar{n}_{\alpha}}{\bar{N}}=\mathrm{e}^{-\varepsilon_{\alpha} / T_{0}}=\mathrm{e}^{-E / 2 T_{0}} .
$$

This is then also the probability of finding two specified particles. They may be the initial (elastic scattering) or some definite other ones, e.g., $p+p \rightarrow \mathrm{A}+\mathrm{B}$. Between Eq. (19.30) and the differential cross-section come, of course, some further considerations (flux factors, centrality condition, influence of the actual masses of A and B), which have been treated in another paper [4]. The main point is that, if we compare Eq. (19.30) with the numerical result of Eq. (19.2) which fits the observed large angle scattering well [4], we find that $T_{0}$ should have a value such that $1 / T_{0}=6.2$. Thus in this case,

$$
T_{0}=1.1 m_{\pi}=151 \mathrm{MeV} .
$$

\footnotetext{
${ }^{6}$ This is in fact the experimental situation [11].
} 
This agrees well with our postulate Eq. (19.24). One could argue that this is a lucky accident, but then it will be hard to explain how this accident leads to a formula which fits the experiment well in a region where the observed cross-section varies over five orders of magnitude [4]. There are in fact not many formulas of physics which cover such a range.

The probability of finding two specified particles [see Eq. (19.30)] is indeed very different from that of finding any two particles: $W(2)=\left(3 T_{0} / E\right) \mathrm{e}^{-6 T_{0} / E}$. The obvious interpretation is that there is a large number of two-body final states, each with a probability of order $\mathrm{e}^{-2 T_{0} / E}$, ranging from elastic scattering to two heavy fireballs, all contributing to $W(2)$. One can even estimate the number of two-body final states. It is of the order

$$
n(2) \approx \mathrm{e}^{E / 2 T_{0}} W(2)=\frac{3 T_{0}}{E} \mathrm{e}^{E / 2 T_{0}-6 T_{0} / E} .
$$

Putting $E=5.6$, i.e., the c.m. energy minus 2 in a $25 \mathrm{GeV} p p$ collision, one finds $n(2) \approx 3 \times 10^{6}$. This would mean that between the pion ${ }^{7}$ and the heaviest (here possible) fireball ( $\Delta E \approx 5.4$ ) lie some $3 \times 10^{6}$ different states. (The question of the mass spectrum of fireballs is treated below see page p. 200.) Similar considerations will apply to other few-particle channels, and seen from this angle, it no longer seems surprising that $W(2) \approx W(3) \approx W(4) \approx \ldots$.

We now turn to the energy spectrum in general. The density of states in the volume $V$ was, in our units,

$$
g(\varepsilon)=\frac{V \varepsilon^{2}}{2 \pi^{2}}=\frac{\varepsilon^{2}}{2 T_{0}^{2}},
$$

and the number of particles to be expected in the level $\varepsilon_{\alpha}$ was given in Eq. (19.28). The number of particles between $\varepsilon$ and $\varepsilon+\mathrm{d} \varepsilon$ then becomes

$$
w(\varepsilon) \mathrm{d} \varepsilon=\frac{E}{6 T_{0}^{4}} \varepsilon^{2} \mathrm{e}^{-\varepsilon / T_{0}} \mathrm{~d} \varepsilon .
$$

We do not believe that the normalization factor $E / 6 T_{0}^{4}$, which makes $\int w(\varepsilon) \mathrm{d} \varepsilon=$ $\bar{N}=E / 3 T_{0}$, is very meaningful because $\bar{N}$ itself is to be rather different in a more realistic model. We simply write

$$
w(\varepsilon) \mathrm{d} \varepsilon \sim \varepsilon^{2} \mathrm{e}^{-\varepsilon / T_{0}} \mathrm{~d} \varepsilon
$$

Remembering that we put $m=0$, we may as well replace $\varepsilon$ by $p$, the momentum. Then Eq. (19.33) reads

$$
w(p) \mathrm{d} p \sim p^{2} \mathrm{e}^{-p / T_{0}} \mathrm{~d} p
$$

\footnotetext{
${ }^{7}$ Since $p+p \rightarrow$ fireball $+\pi$ leads to the smallest mass $\left(m_{\pi}\right)$ of one of the final particles.
} 
or, if we assume an isotropic distribution (as, so far, we are obliged to), we obtain

$$
w(\mathbf{p}) \mathrm{d}^{3} p \sim \mathrm{e}^{-|\mathbf{p}| / T_{0}} \mathrm{~d}^{3} p
$$

It is a remarkable fact that a formula of this type applies apparently to all highenergy processes, if only we replace $|\mathbf{p}|$ by the transverse momentum $p_{\perp}=|\mathbf{p}| \sin \theta$. Let us simply do that and leave the question of how to justify it and get rid of the isotropy to later speculations. Then, if we write

$$
w\left(p_{\perp}\right) \sim \mathrm{e}^{-p_{\perp} / T_{0}},
$$

conservation of total energy could be left to the longitudinal component. Thus Eq. (19.36) would hold true whatever the actual number of particles is. We would expect such a law to govern, not only processes like $p+p \rightarrow \mathrm{A}+\mathrm{B}$, but also the transverse momentum distribution of the many particles produced in cosmic ray jets. Our model would thus explain the hitherto obscure fact that experimentally the transverse momentum distribution in high-energy events is independent not only of the primary energy, but even of the number of particles involved. Since $T_{0}$ depends only on the range of interaction, cosmic ray jets and large angle scattering must show the same behaviour There might, of course, be some slowly varying factors (powers of $p$ and/or $E$ ) in front of the exponential which differ from case to case, but the asymptotic behaviour should be dominated by Eq. (19.36). Orear [14] points out that Eq. (19.36) is a rather good fit to many processes. He quotes experimental results on $p p$ elastic scattering, $p+p \rightarrow \pi+d, \pi+p \rightarrow \pi+p$, and finds that they are all well fitted [the $\pi+p$ data are rather meagre and can only be said not to disagree with Eq. (19.36)] by our Eq. (19.36) if one takes $T_{0}=158 \mathrm{MeV}$ ( $p p$ elastic), $160 \mathrm{MeV}(p+p \rightarrow \pi+d)$.

Figure 19.2 may illustrate how good the fit actually is. Orear (from whose paper [14] the figure is taken) plots $E^{2} \mathrm{~d} \sigma_{\mathrm{el}} /\left.\mathrm{d} \omega\right|_{\mathrm{pp}}$ as a function of $p_{\perp}=p \sin \theta$. The fit

$$
\left.E^{2} \frac{\mathrm{d} \sigma}{\mathrm{d} \omega}\right|_{\mathrm{pp}}=\text { const. } \times \mathrm{e}^{-p_{\perp} / T_{0}}, \quad T_{0}=158 \mathrm{MeV},
$$

is really excellent if one keeps in mind that it covers a range of the primary (lab) momentum between $p_{0}=1.7 \mathrm{GeV} / c$ and $p_{0}=30.7 \mathrm{GeV} / c$ and a range of $\mathrm{d} \sigma / \mathrm{d} \omega$ of eight powers of 10 . As an aside, we mention that the factor $E^{2}$, which seems necessary to make the fit so good, is predicted from a simple 'centrality condition' and is contained in the formula for large angle elastic and exchange scattering recently proposed by the present author [4].

The above values of $T_{0}$ agree well with our postulate $T \approx m_{\pi}$. Furthermore, Cocconi, Koester, and Perkins [15] and Fowler and Perkins [11] find from high energy nucleon-nucleon collisions that the transverse momentum distribution of pions is given by Eq. (19.36) with an apparent value of $T_{0} \approx 170 \mathrm{MeV}$, a somewhat broader distribution than the one with $T_{0} \approx 150 \mathrm{MeV}$. This broadening is to 


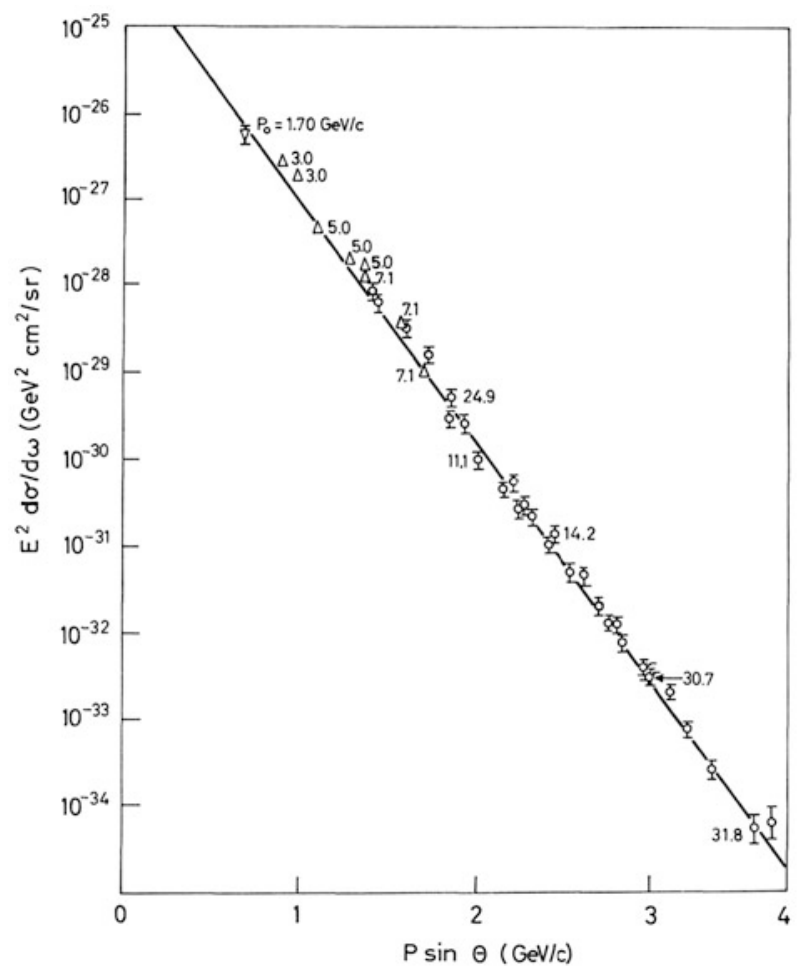

Fig. 19.2 $E^{2} \mathrm{~d} \sigma_{\mathrm{el}} /\left.\mathrm{d} \omega\right|_{\mathrm{pp}}$ as a function of the transverse momentum. Taken from [14]

be expected if we remember that the pions observed experimentally are not the particles produced in the first instant: they are the end-products of a chain of decays, each of which is governed by a law like Eq. (19.36), and the broadening is simply a kinematical effect. If one wishes to calculate this effect quantitatively, then one must no longer put the masses equal to zero. A very simple example is carried through in Appendix 2. We assume that a fireball of mass $m^{*} \gg T_{0}$ emits a particle of mass $m_{\ell}$ (not $\gg T_{0}$ ) and we consider only one-dimensional (transverse) motion: the momentum distribution of $m^{*}$ in the c.m. system is $w\left(p^{*}\right)=\exp \left(-\varepsilon^{*} / T_{0}\right)$, while that of $m_{\ell}$ in the rest frame of the fireball is $w\left(p_{\ell}\right)=\exp \left(-\varepsilon_{\ell} / T_{0}\right)$. Then the momentum distribution of the emitted lighter particle in the c.m. frame becomes $W(p) \approx$ const. $\times\left(\varepsilon+m^{*}\right)^{-1 / 2} \exp \left(-\varepsilon / T_{\text {eff }}\right)$, where $T_{\text {eff }}$ increases monotonically and slowly with $\varepsilon$ : for $\varepsilon=m_{\ell}$, it equals $T_{0}$ and for $\varepsilon \rightarrow \infty$, it reaches $2 T_{0}$. For a fireball of mass $m^{*}=1$ and for $\varepsilon \approx p=1 \mathrm{GeV}$, one finds $T_{\mathrm{eff}} \approx 4 T_{0} / 3$, and a fireball with $m^{*}=2$ would, for the same $\varepsilon$, lead to $T_{\text {eff }} \approx 6 T_{0} / 5$.

We stress once more (see Appendix 1$)$ that, although the relative fluctuation $\left(\overline{N^{2}}-\right.$ $\left.\bar{N}^{2}\right) / \bar{N}^{2}$ tends to one, this is not so for $\left(\overline{w^{2}}-\bar{w}^{2}\right) / \bar{w}^{2}$. In other words, notwithstanding the flatness of the distribution of multiplicities, we should find a sharp distribution 


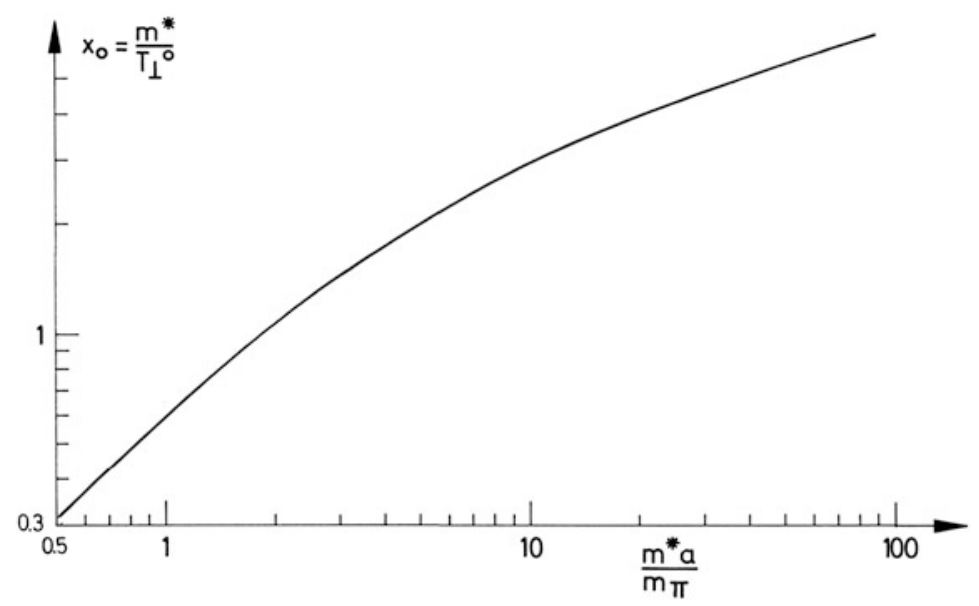

Fig. 19.3 Relation between the mass $m^{*}$ and the temperature $T_{\perp}^{0}$

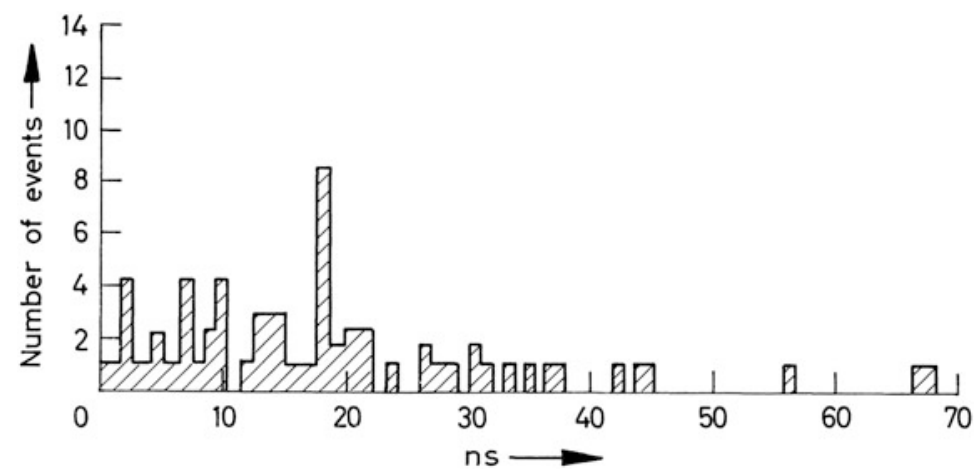

Fig. 19.4 Experimental distribution of multiplicities in 64 events at primary energies $6 \times 10^{3} \leq$ $E_{p} \leq 4 \times 10^{4} \mathrm{GeV}$. Taken from [11]

about the predicted momentum spectrum. It is instructive to look at the experimental Figs. 19.3 and 19.4. They show the sharp distribution about the predicted spectrum and the flat distribution of multiplicities.

We conclude this section by admitting that our reinterpretation of the original model was not always very convincing. In particular, the step from the energy distribution $\bar{n}=\mathrm{e}^{-\varepsilon_{\alpha} / T_{0}}$ to the transverse momentum distribution $w\left(p_{\perp}\right) \sim \mathrm{e}^{-p_{\perp} / T_{0}}$ is mainly suggested by the experimental evidence. But even with these inconsistencies in mind, we believe that the model contains some truth. We shall now try to strengthen this optimism by some speculations about possible improvements. 


\subsection{Speculations on a More Realistic Model}

Our model of massless distinguishable particles in an energy independent volume $V$ was worked out in such detail because of its simplicity. We shall now try to improve the model. Convincing improvements have not yet been carried through. Only an essay will be given. We discuss in turn:

- Angular distribution and multiplicity, see p. 200

- The case of nonzero masses, see p. 206

- A speculation on the mass spectrum of fireballs, see p. 209

- Elastic (and exchange) scattering, see p. 210

- A logical difficulty of the model, see p. 213

\section{Angular Distribution and Multiplicity}

Fermi, in his paper initiating the statistical model [1], consider the possibility of a Lorentz contracted interaction volume. Assuming such a volume, we would already obtain from the uncertainty relation a suggestion of the character of the momentum distribution: the spherical volume $V=(4 \pi / 3)\left(1 / m_{\pi}\right)^{3}$ would become a flat rotational ellipsoid with a transverse half-axis of length $1 / m_{\pi}$ and a longitudinal (with respect to the collision axis) half-axis of length $1 / \gamma m_{\pi}$, where $\gamma=\left(1-\beta_{\mathrm{cm}}^{2}\right)^{-1 / 2}(=E / 2$ for $p p$ collisions $)$. The uncertainty relation requires a particle which has been kept in such a volume and which is suddenly set free to have momenta of the order of $p_{\perp} \approx m_{\pi} / 2, p_{\perp} \approx \gamma m_{\pi} / 2$. We would expect a similar effect for our model. However, one sees immediately that merely Lorentz contracting this interaction volume will have no other result than to replace $V$ in all our formulas by $V / \gamma$ and consequently $T_{0}$ by $T_{0} \gamma^{1 / 3}$. In such a contracted volume, the energy levels will be of the type

$$
\varepsilon_{\alpha}=c \sqrt{\alpha_{2}^{2}+\alpha_{1}^{2}+\gamma^{2} \alpha_{3}^{2}}, \quad \alpha_{1,2,3}=0, \pm 1, \pm 2, \ldots,
$$

where $c=m_{\pi}\left(6 \pi^{2}\right)^{1 / 3}$ for a rectangular box of volume

$$
l_{1} l_{2} \frac{l_{3}}{\gamma}=\frac{1}{\gamma} \frac{4 \pi}{3}\left(\frac{1}{m_{\pi}}\right)^{3},
$$

and our $z=\sum \mathrm{e}^{-\varepsilon_{\alpha} / T}$ becomes as usual

$$
z(\gamma, T)=\int \mathrm{d} \alpha_{1} \mathrm{~d} \alpha_{2} \mathrm{~d} \alpha_{3} \exp \left(-\frac{C}{T} \sqrt{\alpha_{2}^{2}+\alpha_{1}^{2}+\gamma^{2} \alpha_{3}^{2}}\right)=\frac{V_{0}}{\gamma \pi^{2}} T^{3},
$$


where $V_{0}=4 \pi /\left(3 m_{\pi}^{3}\right)$. From here, all the machinery of Sect. 19.2 runs as before, only $V_{0} / \gamma$ replaces $V$ everywhere. Therefore, not only is nothing gained from the angular distribution, but we even have to accept the unpleasant fact (in view of the experimental findings) that $T_{0}$ would no longer be constant $\approx 150 \mathrm{MeV}$, but instead would increase with energy:

$$
T_{0}=\left(\frac{E}{2}\right)^{1 / 3}\left(\frac{\pi^{2}}{V_{0}}\right)^{1 / 3} \approx\left(\frac{E}{2}\right)^{1 / 3} \times 150 \mathrm{MeV} .
$$

In order to overcome this difficulty, we now make the drastic assumption that the longitudinal and transverse motion can be treated independently (we shall try afterwards to give it a shade of justification). In that case, we again consider a rectangular box with volume $V_{0} / \gamma$, where $V_{0} \approx(4 \pi / 3)\left(1 / m_{\pi}\right)^{3}$ is our old $V$. We choose the sides to be

$$
l_{1}=l_{2}=V_{0}^{1 / 3}, \quad l_{3}=\frac{1}{\gamma} V_{0}^{1 / 3} .
$$

Then we have two independent problems of statistical thermodynamics, viz.,

- in a one-dimensional volume $V_{\|}=V_{0}^{1 / 3} / \gamma$ (longitudinal),

- in a two-dimensional volume $V_{\perp}=V_{0}^{2 / 3}$ (transverse).

The densities of the energy levels become in our units

$$
\begin{aligned}
& g_{\|}\left(p_{\|}\right) \mathrm{d} p_{\|}=\frac{V_{\|} \mathrm{d} p_{\|}}{h}=\frac{V_{0}^{1 / 3}}{2 \pi \gamma} \mathrm{d} p_{\|}, \\
& g_{\perp}\left(p_{\perp}\right) \mathrm{d} p_{\perp}=\frac{V_{\perp} 2 \pi p_{\perp} \mathrm{d} p_{\perp}}{h^{2}}=\frac{V_{0}^{2 / 3}}{2 \pi} p_{\perp} \mathrm{d} p_{\perp},
\end{aligned}
$$

and with

$$
z=\sum_{\alpha} \mathrm{e}^{-\varepsilon_{\alpha} / T} \longrightarrow \int g(p) \mathrm{e}^{-p / T} \mathrm{~d} p,
$$

we obtain

$$
\begin{array}{ll}
z_{\|}=\frac{V_{0}^{1 / 3} T_{\|}}{2 \pi \gamma}, & Z_{\|}=\frac{1}{1-z_{\|}} \text {diverges for } T_{\|}^{0}=\frac{2 \pi \gamma}{V_{0}^{1 / 3}}=2 \gamma \pi^{1 / 3} T_{0}, \\
z_{\perp}=\frac{V_{0}^{2 / 3} T_{\perp}^{2}}{2 \pi}, & Z_{\perp}=\frac{1}{1-z_{\perp}} \text { diverges for } T_{\perp}^{0}=\frac{\sqrt{2 \pi}}{V_{0}^{1 / 3}}=\sqrt{2 \pi^{-1 / 3}} T_{0} .
\end{array}
$$

As we decided to treat the longitudinal and the transverse motion independently, there is no reason to insist that the two temperatures associated with these motions 
should be equal. Since our old $T_{0}$ equals $\left(\pi^{2} / V_{0}\right)^{1 / 3}$, we see that $T_{\perp}^{0} \approx T_{0}$ and $T_{\|} \approx \gamma 3 T_{0}$. To every high energy collision in which both energies $E_{\|}$and $E_{\perp}$ are large (i.e., where total c.m. energy and momentum transfer are $\gg m_{\pi}$ ), we would again ascribe a temperature $\approx T_{0}$. We would, however, associate this temperature with the transverse motion only and provide another temperature $\approx 3 \gamma T_{0}$ for the longitudinal one.

Is this picture justified? It is well known (see, e.g., [16]) that a consistent description of scattering should employ wave packets-aimed at each other-rather than plane wave states. A plane wave state would be non-localized and $T$ would be zero (it would be zero for every completely non-localized state, whether or not $\mathbf{p}$ is sharp), but a scattering experiment is equivalent to a position measurement of the colliding particles with the high precision of the linear dimensions of the order of $1 / m_{\pi}$. In that case we could ascribe a temperature $T \lesssim T_{0}$ to the localized wave packets before the collision. Let us then make the rather unconventional speculation and imagine that we could - on the basis of our considerations in Sect.19.3ascribe to the (localized) incoming particle (in its rest system) a temperature $T \lesssim T_{0}$, which depends, as we saw, neither on the number of particles in a volume nor on the energy, but only on the volume. The (localized) hadron would then have a 'temperature' $T \lesssim T_{0}$ and only the conservation laws forbid it to radiate off mesons and nucleon-antinucleon pairs, etc. We may think of $T \lesssim T_{0}$ being the temperature of the cloud of virtual particles. The conservation laws would play the role of a box with rather rigid walls in which the virtual particles are enclosed and which they cannot leave. In the rest system of the nucleon, the energy spectrum of these virtual particles is isotropic; for the momentum distribution in the forward direction of the incoming nucleon, one has

$$
w\left(p_{\|}^{*}\right) \sim \mathrm{e}^{-\left|p_{\|}^{*}\right| / T_{0}},
$$

where the star indicates the rest system of the nucleon. [This distribution is very different from that in a Newton-Wigner localized state [17]. This is not surprising as we are dealing with the localized state of a physical particle, whereas the NewtonWigner state describes a bare localized (Klein-Gordon) particle. It may be possible to relate our philosophy to the 'bootstrap' model of hadrons.] Consider those virtual particles which go in the forward half-space. The $p_{\|}^{*}$ will appear in the c.m. system with momentum $p_{\|}=\gamma\left(p_{\|}^{*}+\beta E^{*}\right)$, where $\beta$ is the velocity of the nucleon seen from the c.m. system of the collision. As $\beta \approx 1$ and $E^{*} \gtrsim p_{\|}^{*}$ (still neglecting masses), we have $p_{\|} \approx 2 \gamma p_{\|}^{*}$. Seen from the c.m. system, the momentum distribution of the virtual particles around the incoming nucleons with then be roughly

$$
\begin{aligned}
& w_{\|}\left(p_{\|}\right) \sim \mathrm{e}^{-p_{\|} / 2 \gamma T_{0}}+\text { contribution from backward going particles }, \\
& w_{\perp}\left(p_{\perp}\right) \sim \mathrm{e}^{-p_{\perp} / T_{0}} \quad \text { (unchanged). }
\end{aligned}
$$

The collision, which now takes place, will 'carry out the position measurement' and loosen the constraints imposed by the conservation laws. In other words, the collision breaks the rigid walls of the volume $V$ and the virtual particles can become 
real. They would escape with momentum spectra of the type (19.42), where $w_{\|}\left(p_{\|}\right)$ contributes $\mathrm{e}^{-p_{\|} / 2 \gamma T_{0}}$ to the forward and backward directions-each nucleon to one of these directions. [The contributions from the particles which were emitted backward in the nucleon rest frame have low average momenta and do not follow the distribution $w_{\|}\left(p_{\|}\right)$.]

We see that such a picture would rather naturally lead to an effective longitudinal temperature $T_{\|}$being about $\gamma$ times larger than $T_{\perp}$ (and indeed the two Lorentz contracted incoming particles will, when they get into touch with each other, look like one Lorentz contracted volume at rest in the c.m. system). We only have to assume that, in most cases, the collision time is so short that a thermal equilibrium $\left(T_{\|} \approx T_{\perp}\right)$ cannot be reached. The simple model described in [4] shows that the fraction of collisions in which thermal equilibrium may be reached, is about $1 / \gamma^{2}$ of all inelastic ones. ${ }^{8}$ That means that in roughly $1-1 / \gamma^{2}=\beta^{2} \approx 1$, i.e., in nearly all collisions, the thermal equilibrium is not reached and the longitudinal and transverse temperatures can be different and the volume of interaction looks Lorentz contracted. Then our above treatment would be justified. If, however, in the very few remaining collisions $\left(\sigma \approx \sigma_{\text {inel }} / \gamma^{2}\right)$ thermal equilibrium is (more or less) reached, then we would come back to our model of Sect. 19.2 and we would obtain the unique temperature $T_{\|} \approx T_{\perp} \approx T_{0}$ in a volume which is no longer Lorentz contracted. The emission of particles would have a tendency to become isotropic. The interesting point is, however, that then again the transverse (and, incidentally, the longitudinal) momentum distribution would be described by $\sim \mathrm{e}^{-p_{\perp} / T_{0}}$, since $T_{0}$ does not depend on the amount of energy transferred to the transverse degree of freedom. We thus come to the following conclusion:

Conclusion Whenever in a collision-no matter whether central or not-total energy and momentum transfer are both much larger than $m_{\pi}$, we expect the transverse momentum distribution to be independent of the total energy, of the momentum transfer, and of the particle number, and to be approximately of the form $w\left(p_{\perp}\right) \sim \exp \left(p_{\perp} / T_{0}\right)$. The longitudinal momentum distribution will be roughly of the form $w\left(p_{\|}\right) \sim \exp \left(p_{\|} / \alpha \gamma T_{0}\right), \alpha \approx 1$, except for very central collisions, where it becomes similar to the transverse distribution.

Having succeeded or failed (as the reader may decide) to justify the independent treatment of longitudinal and transverse motion, we go back to Eq. (19.41) and draw a few further conclusions.

First of all, the distributions of particles with $p_{\|}$and $p_{\perp}$ become

$$
\begin{aligned}
& \bar{n}_{\|}\left(p_{\|}\right)=\frac{\bar{N}}{T_{\|}^{0}} \mathrm{e}^{-p_{\|} / T_{\|}^{0}}, \quad T_{\|}^{0} \approx 3 \gamma T_{0}, \\
& \bar{n}_{\perp}\left(p_{\perp}\right)=\frac{\bar{N}}{T_{\perp}^{02}} p_{\perp} \mathrm{e}^{-p_{\perp} / T_{\perp}^{0}}, \quad T_{\perp}^{0} \approx T_{0} \approx m_{\pi},
\end{aligned}
$$

\footnotetext{
${ }^{8}$ There is of course a continuous range of intermediate situations between 'central' and 'peripheral'.
} 
which is just what we said in the above conclusion. Of course, we cannot expect these two independent distributions to be exact in cases of low multiplicity, where energy-momentum conservation imposes severe restrictions. In particular, in large angle elastic and exchange scattering, the transverse momentum distribution uniquely fixes the longitudinal one. It remains to explain why, when at most one of the two given distributions can hold, nature apparently chooses the transverse one (for evidence, see Fig. 19.2). In cases of large multiplicity, the two distributions describe a 'jet'. It is most interesting to learn from experiments [11, 15] that our formula actually fits ${ }^{9}$ the transverse momentum distribution in jets up to at least $p_{\perp} \approx 1.2 \mathrm{GeV} / c$ and for primary energies between $25 \mathrm{GeV}$ and $10^{6} \mathrm{GeV}$ with the one constant $T_{0}$ value of $\approx 160 \mathrm{MeV}$.

Although we decoupled the two directions of motion, we should require that the mean particle number be the same in both systems since we wish after all to describe actual events (in which of course the particles having longitudinal momentum components are just the same as those which have transverse ones). Since from Eq. (19.15) we have $\bar{N}=z /(1-z)$, it follows that

$$
\bar{N}=\frac{z_{\|}}{1-z_{\|}}=\frac{z_{\perp}}{1-z_{\perp}}, \quad z_{\|}=z_{\perp},
$$

and consequently, the relation between the temperatures is

$$
T_{\|}=\gamma V_{0}^{1 / 3} T_{\perp}^{2},
$$

which (only) for $T \rightarrow T_{0}$ can be written

$$
T_{\|}^{0}=\gamma \sqrt{2 \pi} T_{\perp}^{0} .
$$

Next let us consider energy conservation. We can write down the energies contained in the longitudinal and transverse motion, respectively (remembering that $m=0$ ):

$$
\begin{aligned}
& \bar{E}_{\|}=\int p_{\|} \bar{n}_{\|}\left(p_{\|}\right) \mathrm{d} p_{\|}=\bar{N} T_{\|}^{0} \approx 3 \bar{N} \gamma m_{\pi}, \\
& \bar{E}_{\perp}=\int p_{\perp} \bar{n}_{\perp}\left(p_{\perp}\right) \mathrm{d} p_{\perp}=2 \bar{N} T_{\perp}^{0} \approx 2 \bar{N} m_{\pi} .
\end{aligned}
$$

But is the total energy $E$ the sum of these two? We might say so if the two belonged to two really independent systems - but it is just energy-momentum conservation which makes them not completely independent. We could think of defining the total energy by

$$
\frac{1}{\bar{N}} \int \sqrt{p_{\perp}^{2}+p_{\|}^{2}} \bar{n}_{\perp}\left(p_{\perp}\right) \bar{n}_{\|}\left(p_{\|}\right) \mathrm{d} p_{\perp} \mathrm{d} p_{\|},
$$

\footnotetext{
${ }^{9} \mathrm{An}$ apparent slow increase in $T_{0}$ with primary energy can be understood as a kinematical effect (see Appendix 2).
} 
where the correct energy for a particle with $p=\left(p_{\perp}, p_{\|}\right)$is now summed up. But again, multiplying the two, supposedly independent, distributions does not yield the correct distribution $\bar{n}\left(p_{\perp}, p_{\|}\right)$of the particles: it may only tend to the correct one for large multiplicities. We may leave the details and use the fact that, for large $E$ (in $p p$ collisions $\gamma=E / 2$ ), Eq. (19.42) shows that almost all energy is contained in the longitudinal component, which contains $\gamma$ times more than the transverse one. We thus identify $\bar{E}_{\|}$with $E$ for $E$ very large $(\gamma \gg 1$ means $E \gg 1$, that is, much more than our usual condition $E \gg m_{\pi}$ ). We obtain

$$
E \approx 3 \bar{N} \gamma m_{\pi}
$$

and therefore,

$$
\bar{N} \approx \frac{E}{3 \gamma m_{\pi}} \quad\left(=\frac{2}{3 m_{\pi}} \approx 5 \text { for } p p \text { collision }\right) .
$$

This low and energy independent multiplicity does not of course concern the final one of pions, etc. In fact, $\bar{N}$ is the average number of more or less excited particles formed in the first instant (resonances and/or fireballs) which afterwards decay. The actual increase in multiplicities, which is experimentally observed, must be interpreted as an increase in the excitation of the fireballs. The energy independence of $\bar{N}$ and $T_{\perp}^{0}$ implies immediately [by Eq. (19.47)] that the average kinetic energy stored in the transverse motion is itself independent of the primary energy [although strongly fluctuating from event to event, see Eq. (19.19)].

Since the probability of finding just $N$ particles is

$$
W(N)=\frac{z^{N}}{\sum z^{N}}, \quad z=z_{\|}=z_{\perp},
$$

and since $z_{\|} \rightarrow 1$ for $T_{\|} \rightarrow T_{\|}^{0}$, it follows that here also all $N$ values become equally probable in the limit (in such a way, however, that $\bar{N} \rightarrow \approx 5$ ). Although the average number of fireballs is $\approx 5$, the actual number can therefore hardly be predicted. The introduction of masses will, of course, suppress very large $N$ values. Nevertheless, even then we have to expect enormous fluctuations in the multiplicities of final particles produced in collisions at (fixed!) high energy. ${ }^{10}$

Although the present treatment of the problem of the angular distribution is certainly not yet fully correct, it may indicate the direction in which one has to go. Nevertheless, we find quite satisfying results:

- The transverse momentum distribution is independent of:

\footnotetext{
${ }^{10}$ This is actually found [11] at cosmic ray energies: the r.m.s. fluctuation in multiplicity is $\approx \bar{n}_{\mathrm{s}}$ (where $\bar{n}_{\mathrm{s}}$ is the charged multiplicity) (see Fig. 19.4). From the experimental angular distribution follow large fluctuations in the number of fireballs.
} 
- the total energy,

- the number of particles,

- the centrality of the collision,

and always has the form $\sim \exp \left(-p_{\perp} / T_{0}\right), T_{0} \approx m_{\pi}$ (vanishing fluctuations). This holds whenever the total energy and momentum transfer are both $\gg m_{\pi}$. In that case, thermal equilibrium may or may not be attained. It is irrelevant.

- Almost all the energy is contained as kinetic energy in the longitudinal component (jet) and the (strongly fluctuating) transverse energy is on average independent of the primary energy.

- The multiplicity of the first generation of particles (fireballs) fluctuates strongly but is not large. The average value is of order 5 and independent of the primary energy. The strongly fluctuating multiplicity of the last generation (final pions, nucleons, hyperons) will on average increase very slowly, as most of the total energy is contained in the kinetic energy of the fireballs and only a little in their excitation.

These rather-for a statistical model-unusual features explain also in a most natural way why the conventional (Fermi) statistical model of particle production works so much better than one could (in view of the rareness of central collisions) reasonably expect: the mere existence of the 'highest temperature' $T_{0}$ guarantees, so to speak, an everlasting pre-established thermal equilibrium inside the incoming (localized) particles-the collision itself has only to break off the volumes (= conservation laws) in which the clouds were enclosed. Even in noncentral collisions, this pre-established thermal distribution reveals itself, namely in the transverse momenta. Central collisions only help to transfer longitudinal energy into the transverse motion, without effect for the latter, except for an increase in multiplicity. Only in most central collisions can a thermal equilibrium be obtained in the usual sense, and then $T_{\|}^{0} \approx T_{\perp}^{0}$.

\section{The Case of Nonzero Mass}

We return to Eq. (19.41) and remark that with $\gamma=1$, i.e., for a cubic box, we obtain $T_{\|}^{0}=\pi^{1 / 3} 2 T_{0}$ and $T_{\perp}^{0}=\sqrt{2 / \pi^{1 / 3}} T_{0}$, where $T_{0}$ is the value for the threedimensional problem in the same volume. Since $\sqrt{2 / \pi^{1 / 3}}=1.17$, we see that $T_{\perp}^{0} \approx T_{0}$. We also expect the two temperatures to be nearly equal in the case $m \neq 0$. We shall therefore discuss mainly $T_{\perp}^{0}$.

We first consider briefly $T_{\|}^{0}$ and then in more detail $T_{\perp}^{0}$, assuming now that all particles have the same mass $m^{*}$ (to be thought of as the average mass):

1. For the longitudinal component, we have

$$
z\left(m^{*}, T\right)=\sum_{\alpha} \mathrm{e}^{-\varepsilon_{\alpha} / T} \longrightarrow \int g(p) \mathrm{e}^{-\sqrt{p^{2}+m^{* 2}} / T} \mathrm{~d} p
$$


Then $g_{\|}\left(p_{\|}\right)$taken from Eq. (19.40) gives

$$
\begin{aligned}
z_{\|}\left(m^{*}, T\right) & =\frac{V_{0}^{1 / 3}}{2 \pi \gamma} \int_{0}^{\infty} \mathrm{e}^{-\sqrt{p^{2}+m^{* 2}} / T} \mathrm{~d} p \\
& \left.=\frac{V_{0}^{1 / 3} m^{*}}{2 \pi \gamma} \int_{0}^{\infty} \mathrm{e}^{-m^{*} \sqrt{1+x^{2}} / T} \mathrm{~d} x \quad \text { (put } x=\sinh y\right) \\
& =\frac{V_{0}^{1 / 3} m^{*}}{2 \pi \gamma}\left[-\frac{\mathrm{d} K_{0}(\tau)}{\mathrm{d} \tau}\right]_{\tau=m^{*} / T} .
\end{aligned}
$$

The condition that $Z_{\|}$diverges is that $z_{\|}=\uparrow$. Hence

$$
\frac{2 \pi}{m^{*} V_{0}^{1 / 2}} \gamma=-\left.\frac{\mathrm{d} K_{0}(\tau)}{\mathrm{d} \tau}\right|_{\tau=m^{*} / T_{\|}^{0}}=K_{1}\left(m^{*} / T_{\|}^{0}\right) \text {. }
$$

Now the Bessel function $K_{1}(x)$ is a smooth, steadily decreasing function of $x$, with the asymptotic behaviour

$$
K_{1}(x) \longrightarrow \begin{cases}\sqrt{\frac{2}{\pi x}} \mathrm{e}^{-x}, & x \rightarrow \infty \\ \frac{1}{x}, & x \rightarrow 0\end{cases}
$$

Since the left-hand side of Eq. (19.52) goes to $\infty$ when $E \rightarrow \infty$, we conclude that $x \rightarrow 0$. Then we use the second line of Eq. (19.53) to obtain, for $E \rightarrow \infty$,

$$
\frac{2 \pi}{m^{*} V_{0}^{1 / 3}} \gamma=\frac{T_{\|}^{0}}{m^{*}}, \quad T_{\|}^{0}=2 \gamma \pi^{1 / 3} T_{0} .
$$

This agrees with the value found for $m^{*}=0$, as expected. In the longitudinal component, the mass of a fireball is practically always negligible compared to its momentum.

2. The transverse component is treated similarly. We arrive at

$$
\begin{aligned}
z_{\perp}\left(m^{*}, T\right) & =\frac{V_{0}^{2 / 3}}{2 \pi} \int_{0}^{\infty} p \mathrm{e}^{-\sqrt{p^{2}+m^{* 2}} / T} \mathrm{~d} p \\
& =\frac{V_{0}^{2 / 3} m^{* 2}}{2 \pi} \int_{0}^{\infty} x \mathrm{e}^{-m^{*} \sqrt{1+x^{2}} / T} \mathrm{~d} x \\
& =\frac{V_{0}^{2 / 3} T^{2}}{2 \pi}\left(1+\frac{m^{*}}{T}\right) \mathrm{e}^{-m^{*} / T},
\end{aligned}
$$


and the highest temperature $T_{\perp}^{0}$ is defined implicitly by

$$
1=\frac{V_{0}^{2 / 3} T_{\perp}^{0 \ell}}{2 \pi}\left(1+\frac{m^{*}}{T_{\perp}^{0}}\right) \mathrm{e}^{-m^{*} / T_{\perp}^{0}}
$$

The numerical value of $T_{\perp}^{0}$ is not very relevant, because we are largely uncertain about the value of $m^{*}$ [= average of masses produced at the given energy $=$ very slowly varying function of the energy (?)] to be put in. Indeed, we should not use a single value for $m^{*}$ but rather a mass spectrum (see below). What is relevant is that the $m^{*} \neq 0$ case does not change the basic fact that a highest temperature exists. Therefore, all our conclusions drawn so far remain at least qualitatively valid.

Let us nevertheless make a little numerical analysis of Eq. (19.56). For the average mass of 'fireballs', we may expect a value of perhaps the nucleon mass. This is in accordance with our observation that most of all energy in jets must be contained in the kinetic energy of the longitudinal component and only a little in the excitation (= mass) of fireballs. We rewrite Eq. (19.56), putting $x_{0}=m^{*} / T_{\perp}^{0}$ and $V_{0}=(4 \pi / 3)\left(a / m_{\pi}\right)^{3}$ :

$$
\frac{1}{2 \pi}\left(\frac{4 \pi}{3}\right)^{2 / 3}\left(\frac{m^{*} a}{m_{\pi}}\right)^{2}=\frac{x_{0}^{2}}{1+x_{0}} \mathrm{e}^{x_{0}} .
$$

In Fig. 19.3 we plot

$$
\frac{m^{*} a}{m_{\pi}}=\sqrt{2 \pi}\left(\frac{3}{4 \pi}\right)^{1 / 3} \frac{x_{0} \mathrm{e}^{x_{0} / 2}}{\sqrt{1+x_{0}}}
$$

as a function of $x_{0}$.

Put $m^{*}=1$ and $T_{\perp}^{0}=170 \mathrm{MeV}$ so that $x_{0} \approx 6$. We read off

$$
\frac{m^{*} a}{m_{\pi}}=\frac{a}{m_{\pi}} \approx 60,
$$

or $a \approx 9$. This is certainly disappointing. It would mean that the volume in which interaction still takes place would have linear dimensions of the order of nine times the pion Compton wavelength.

It seems, however, that there is a way out, maybe even two ways. The value $T_{\perp}^{0}$ which we inserted is taken from the fit of $\mathrm{e}^{-p_{\perp} / T_{\perp}^{0}}$ to experiments. Taking the masses to be nonzero, one should not expect $p_{\perp}$ in the Boltzmann law, but rather $\sqrt{p_{\perp}^{2}+m^{2}}$. Let $p_{\perp}$ be of the order $m_{\pi}$ and consider the pion transverse momentum ( $m=m_{\pi}$ in the Boltzmann law). Then roughly $\sqrt{p_{\perp}^{2}+m_{\pi}^{2}} \approx \sqrt{2} p_{\perp}$. If one fitted the experiments with $\exp \left(-\sqrt{p_{\perp}^{2}+m_{\pi}^{2}} / T\right)$ instead of with $\mathrm{e}^{-p_{\perp} / T_{\perp}^{0}}$, 
one would expect a $T$ value which is roughly $\sqrt{2}$ times larger than the old one. Thus, for consistency, we should put $T_{\perp}^{0} \approx 240 \mathrm{MeV} \approx 0.25$. Then $x_{0} \approx 4$ and $a m^{*} / m_{\pi} \approx 20, a \approx 3$ for $m^{*}=1$. Actually, if $m^{*}$ were somewhat smaller than the nucleon mass, say $\approx 0.75$, we would arrive at $x_{0} \approx 3$ and $a m^{*} / m_{\pi}=10, a \approx 2$. This is already quite reasonable.

The other way out of the difficulty may lie in introducing a mass spectrum of excited states.

\section{A Speculation on the Mass Spectrum of 'Fireballs'}

If $\rho\left(m^{*}, T\right) \mathrm{d} m^{*}$ is the mass spectrum, the true $z(T)$ would be given by [see Eq. (19.55)]

$$
z_{\perp}(T)=\int_{0}^{\infty} \rho\left(m^{*}, T\right) z_{\perp}\left(m^{*}, T\right) \mathrm{d} m^{*}=\frac{V_{0}^{2 / 3} T^{2}}{2 \pi} \int_{0}^{\infty} \rho\left(m^{*}, T\right)\left(1+\frac{m^{*}}{T}\right) \mathrm{e}^{-m^{*} / T} \mathrm{~d} m^{*} .
$$

Since under no circumstances can $z_{\perp}(T)$ become large than one, the integral must converge. This puts a limit on the asymptotic behaviour of $\rho\left(m^{*}, T\right)$ :

The mass spectrum of highly excited hadrons (fireballs) must grow less than $\mathrm{e}^{m^{*} / T}$, where

$T$ is of the order of $m_{\pi}$.

That it will indeed grow almost that fast is seen when we consider that $\rho\left(m^{*}, T\right) \mathrm{d} m^{*}$ is the total number of states between $m^{*}$ and $m^{*}+\mathrm{d} m^{*}$ of a 'fireball'. But such a fireball itself is again described by our model-an unspecified number of distinguishable particles in a volume $V_{\mathrm{c}}$ with a total energy $E=m^{*}$. The density of states of such a system is roughly $\mathrm{e}^{S}$, where $S(E, V)$ is the entropy. Since the temperature of the system (at sufficiently high energy) becomes $T_{0}=$ const., it follows that asymptotically [namely when $\left(\log m^{*}\right) / m^{*} \rightarrow 0$ ],

$$
S\left(E, V_{0}\right) \longrightarrow \frac{E}{T_{0}}, \quad \rho\left(m^{*}, T\right) \longrightarrow \mathrm{e}^{m^{*} / T_{0}}
$$

We may then put

$$
\rho\left(m^{*}, T\right) \equiv \frac{1}{T} f\left(m^{*} / T\right) \mathrm{e}^{m^{*} / T},
$$

and obtain for $z_{\perp}=1$,

$$
\frac{2 \pi}{V_{0}^{2 / 3}}=T_{\perp}^{0} \int_{0}^{\infty} f(x)(1+x) \mathrm{d} x
$$


As experimental evidence shows that $T_{\perp}^{0}$ is of the order of $m_{\pi}$, it is required that

$$
\int_{0}^{\infty} f\left(m^{*} / T_{0}\right)\left(1+\frac{m^{*}}{T_{0}}\right) \mathrm{d}\left(m^{*} / T_{0}\right) \approx 1, \quad f\left(m^{*} / T_{0}\right) \equiv T_{0} \rho\left(m^{*}, T_{0}\right) \mathrm{e}^{-m^{*} / T_{0}} .
$$

It is not clear whether Eq. (19.63), which puts a condition on the mass spectrum, is compatible with the fact that the mass spectrum should follow from the theory itself. It could be that this leads to an interesting self-consistency problem with further consequences (a kind of 'bootstrap' at high temperature).

In any case, we see that the introduction of a mass spectrum $\rho\left(m^{*}, T\right)$ may resolve the apparent difficulty in reconciling the numerical value of $T_{\perp}^{0}$, as found experimentally, with the requirement $m^{*} \neq 0$ (and not too small). We may presently at least hope that the value of the integral in Eq. (19.63) is near to one and consequently neither $T_{\perp}^{0}$ nor $V_{0}$ have to have unreasonable values.

\section{Elastic and Exchange Scattering}

Whatever the actual value of $T_{0}$ may turn out to be, we know that it exists. Let us then assume that it is indeed of the order of $m_{\pi}$. Taking the masses seriously and still treating the transverse and longitudinal motion as independent, we expect the transverse momentum distribution to have the form [see Eq. (19.43)]

$$
w\left(p_{\perp}\right) \sim p_{\perp} \mathrm{e}^{-\sqrt{p_{\perp}^{2}+m^{* 2}} / T_{0}}
$$

where strictly speaking $m^{*}$ is the mass of that type of fireball whose distribution we wish to describe. Since actually mostly pions are observed, and these come from a chain of decays, the observed distribution will be somewhat different, and in fact broader (see Appendix 2). But, experimentally [11, 14, 15], it definitely is of the form given in Eq. (19.64) with $T_{0} \approx 160 \mathrm{MeV}$. Experimentally, the $T_{0}$ used to fit the distribution by $p_{\perp} \mathrm{e}^{-p_{\perp} / T_{0}}$, seems to increase (very slowly) with the primary energy [11]. This can be due to the slowly increasing mean excitation of the fireballs which then, on average, decay in a number of steps into the final particles. This number of steps will increase with the mass $m^{*}$ of the fireball. Each of these steps will broaden the spectrum resulting from the preceding decay. If one tries nevertheless to fit with $p_{\perp} \mathrm{e}^{-p_{\perp} / T_{0}}$, then $T_{0}$ must obviously increase somewhat (see Appendix 2).

The situation is different if we apply this formula to elastic scattering of nucleons, for instance. There $m^{*}$ really means the nucleon mass and then the differential elastic cross-section should obey

$$
\frac{\mathrm{d} \sigma_{\mathrm{el}}}{\mathrm{d} w} \sim \mathrm{e}^{-\sqrt{p_{\perp}^{2}+m^{2}} / T_{0}}
$$


There will be kinematical factors in front of this expression, which are not too easily worked out, because conceptually $w\left(p_{\perp}\right)$ and $\mathrm{d} \sigma / \mathrm{d} w$ are somewhat different: $\mathrm{d} \sigma / \mathrm{d} w$ applies to a system with fixed total energy, whereas $w\left(p_{\perp}\right)$ applies to a system where the energy of a single particle is not fixed. Indeed, $w\left(p_{\perp}\right) \mathrm{d} p_{\perp}$ shows how many there might be in the interval given by $\mathrm{d} p_{\perp}$. If we applied this $w\left(p_{\perp}\right)$ literally to elastic scattering, we would then have

$$
\mathrm{d} p_{\perp}=\mathrm{d}(p \sin \theta)=\sin \theta \mathrm{d} p+p \cos \theta \mathrm{d} \theta=p \cos \theta \mathrm{d} \theta,
$$

because $\mathrm{d} p=0$ for fixed total energy. We see then that, for geometrical reasons, $w\left(p_{\perp}\right) \mathrm{d} p_{\perp}=0$ for $\theta=\pi / 2$ and hence that at $90^{\circ}$,

$$
\frac{\mathrm{d} \sigma_{\mathrm{el}}}{\mathrm{d} w} 2 \pi \sin \theta \mathrm{d} \theta \neq w\left(p_{\perp}\right) \mathrm{d} p_{\perp},
$$

since the left-hand side is nonzero. However, for smaller angles such a formula looks most natural. We may then tentatively simply replace $\mathrm{d} p_{\perp}$ by $p \mathrm{~d} \theta$, which somehow compensates for our disregarding the strong correlations between longitudinal and transverse distributions in a two-body case. Then, using

$$
w\left(p_{\perp}\right)=\text { const. } \times p_{\perp} \mathrm{e}^{-\sqrt{p_{\perp}^{2}+m^{2}} / T_{0}},
$$

the result is ${ }^{11}$

$$
\frac{\mathrm{d} \sigma_{\mathrm{el}}}{\mathrm{d} w}=\text { const. } \times p^{2} \mathrm{e}^{-\sqrt{p_{\perp}^{2}+m^{2}} / T_{0}} \quad(\text { angles not near } \pi / 2),
$$

whereas for angles near $90^{\circ}$, some other factor should replace $p^{2}$.

This other factor may involve an extra condition: centrality. The point is this: the distribution $w\left(p_{\perp}\right)$ is, as we saw, independent of the extent to which thermal equilibrium (between longitudinal and transverse motion) is reached. This holds certainly only for systems of many particles where a large value of $p_{\perp}$ does not then require the collision to have been central. In the two-body case a large value $p_{\perp} \rightarrow$ $p$ implies a central collision and consequently we expect this further condition to modify $w\left(p_{\perp}\right)$. As shown in [4], the centrality condition can be taken into account by multiplying the relevant total inelastic cross-section by $1 / \gamma^{2}=4 / E^{2}$. Then, for larger angles, we should expect

$$
\frac{\mathrm{d} \sigma_{\mathrm{el}}}{\mathrm{d} w} \approx \text { const. } \times \mathrm{e}^{-\sqrt{p_{\perp}^{2}+m^{2}} / T_{0}} \underset{\text { near } 90^{\circ}}{\longrightarrow} \text { const. } \times \mathrm{e}^{-p_{\perp} / T_{0}} .
$$

\footnotetext{
${ }^{11}$ Note that $\mathrm{d} \sigma / \mathrm{d} w$ is a function of the two independent variables $E$ and $\theta$. Our formulas (19.68) and (19.69) claim to describe the differential elastic cross-section as a function of both of these variables.
} 
Perhaps the 'constant' still varies with $E$. In fact, the best fit is obtained by putting it equal to $1 / E^{2}$ (see Fig. 19.2).

The most interesting property of our new formula is that, for $p_{\perp} \rightarrow 0$ (forward direction), it gives

$$
\frac{\mathrm{d} \sigma_{\mathrm{el}}}{\mathrm{d} w} \approx \text { const. } \times p^{2} \exp \left(-\frac{1}{T_{0}} \frac{p_{\perp}^{2}}{2 m}\right) \underset{\text { near } 0^{\circ}}{\longrightarrow} \text { const. } \times p^{2},
$$

which is required by the optical theorem together with the empirical facts that the scattering amplitude tends to become purely imaginary and $\sigma_{\text {tot }} \rightarrow$ const. Even the functional form coincides with that of the observed diffraction peak-for small angles, $p^{2} \rightarrow-t$ (invariant square of the momentum transfer) and Eq. (19.70) reads

$$
\left.\frac{\mathrm{d} \sigma}{\mathrm{d} t}\right|_{t \rightarrow 0}=\text { const. } \times \mathrm{e}^{t / 2 m T_{0}} .
$$

Experimentally, it is of the form $\mathrm{e}^{+b t}$ with $b \approx 10 \mathrm{GeV}^{-2}$. However, if $T_{0}$ were of the order of $m_{\pi} \approx m / 6.8$, this would give $b \approx 3.4 / \mathrm{m}^{2}=3.9 \mathrm{GeV}^{-2}$. This value is much too small. Conversely, in our present model, it would mean that, as $p_{\perp} \rightarrow 0$, a temperature of

$$
T_{0}^{*} \approx 0.4 T_{0}
$$

would be needed to fit the data. Now our model by no means excludes a temperature lower than $T_{0}$ and indeed we cannot even justly require that $T_{0}$ should be reached when the momentum transfer is extremely small. [Remember the conclusion on p. 203.] This may be interpreted as saying that the temperature of that cloud of virtual particles, which constitutes what we call a localized nucleon, is here about one half of $T_{0}$, because at this small momentum transfer, it was not so well localized. The temperature reaches $T_{0}$ only if a sufficient amount of energy is transferred to the transverse motion. (It would be interesting to see what our model has to say about peripheral collisions $p+p \rightarrow \mathrm{N}^{*}+\mathrm{N} \rightarrow \mathrm{N}+\mathrm{N}+\pi$, etc.)

Whatever the reason, as a matter of fact (Orear, private communication),

$$
\frac{\mathrm{d} \sigma_{\mathrm{el}}}{\mathrm{d} w} \sim \mathrm{e}^{-\sqrt{p_{\perp}^{2}+m^{2}} / T_{0}}
$$

seems to fit the experimental data with $T_{0} \approx 50 \mathrm{MeV}$ in the diffraction region and with $T_{0} \approx 150 \mathrm{MeV}$ outside the diffraction region. The temperature required for the fit changes rather rapidly from one value to the other. If one puts

$$
\Delta E_{\perp}=\sqrt{p_{\perp}^{2}+m^{2}}-m
$$


then $\Delta E_{\perp}$ is the energy transferred to the transverse motion. It then happens that, in the diffraction region, $\Delta E_{\perp}<0.3$, and in the region where $T_{0} \approx 150 \mathrm{MeV}$ gives a good fit, $\Delta E_{\perp}>0.7$. Tentatively using Fig. 19.1 with $\bar{E} \equiv \Delta E_{\perp}$, one sees that, in the diffraction region, $T<0.75 T_{0}$, and in the other region, $T>0.9 T_{0}$ would result. This is not of course to be taken too seriously, because in Fig. 19.1 it was supposed that the masses could be neglected. Anyway, the tendency is right.

It is probably not just to demand from our model that it should give even the numerical behaviour in the diffraction region correctly. Indeed, in that region the neglected strong geometrical correlation between the longitudinal and transverse momenta should again become as important as near $90^{\circ}$.

The present remarks are largely just guesswork. It remains to clear up the relation of our model to elastic scattering and peripheral collisions.

\section{A Logical Difficulty of the Model}

We have employed statistical thermodynamics of distinguishable particles. This is strictly speaking inconsistent, since nature certainly does not work this way. Indeed, even if we are right in saying that most contributions come from states in which all particles (resonances, fireballs) are different, there are certainly states in which, for instance, five $\pi^{+}$are already present in the first generation.

To be really consistent, we should have worked out a statistics of, say, $M$ different species of particles. Particles of the same species must then be considered to be indistinguishable (and a statistics, Bose or Fermi, to be prescribed) and the number $N_{i}$ of particles of each kind, as well as the total number $N=\sum N_{i}$ of particles, has to be left open as before. Finally, one lets $M \rightarrow \infty$. One sees immediately that the number of particles of each single kind would then tend to zero and we should come back to our model.

In the case of zero masses one finds, however, that this does not work. One simply obtains $[Z(V, T)]^{M}$, where $Z(V, T)$ is the usual partition function of indistinguishable particles, and in fact that of a massless Fermi or Bose gas, as the case may be. With $M \rightarrow \infty$, everything diverges at any temperature. That is easily understood if one realizes that, for $M \rightarrow \infty$, there is an infinity of states of our gas even if the total number $N$ of particles in it is kept fixed: each single particle may be removed from the gas and be replaced by one of another species. From that operation, a new state with the same energy results, whence the sum over states is infinite.

The situation becomes different if the particles have a mass. Then replacing a particle by one of another species means changing the energy of the system, and one cannot generate an infinity of different states of the same energy. Again, with the number $M$ of kinds of particles going to infinity, one would find that the number of particles of a given kind tends to zero. It is hoped that such a statistics will become equivalent to our present model. This, however, has not yet been worked out. It 
seems to be an urgent problem in view of the success of the present model. Work is in progress.

\subsection{Summary and Conclusions}

Our model uses only three basic experimental facts:

1. The strong interactions are strong enough to produce many resonances and even fireballs. We assume that the latter are only an 'extrapolation' of the resonances to very high energies.

2. The strong interactions have a range of the order of the Compton wavelength of the pion.

3. In high-energy collisions, the duration of contact is in general so short that a thermodynamical equilibrium (in the sense of Fermi's statistical theory) cannot be reached.

From (1) it follows that particles are to be considered as (quasi) distinguishable, while (2) determines the volume in which the system is enclosed, and (3) allows one to treat the longitudinal and transverse motion as (nearly) independent. All the rest is straightforward and simple statistical thermodynamics with the following results:

- A universal highest temperature $T_{0} \approx m_{\pi}$ (corresponding to $\approx 10^{12} \mathrm{~K}$ ) governs all high-energy processes involving strong interactions (and only these; no highest temperature exists for gravitational, weak, and electromagnetic interactions since they do not produce the many resonances which make the particles distinguishable).

- The transverse momentum distribution in high-energy collisions of hadrons is a Boltzmann distribution with constant temperature $T_{0} \approx m_{\pi}$ independent

- of the primary energy $\left(1 \leq E_{\mathrm{lab}} \leq 10^{6} \mathrm{GeV}\right)$,

- of the number of particles involved-for two particles it gives elastic scattering, for many particles the jets,

- of the centrality (= degree of thermal equilibrium) of the collision.

- Almost all energy is contained in the longitudinal component as kinetic energy. Only a small fraction is used for the excitation of fireballs. The total transverse energy fluctuates strongly but is on average practically independent of the primary energy.

- The multiplicities of particles produced fluctuate strongly (dispersion of order 1) about a slowly increasing mean value, whereas the fluctuations about the Boltzmann law for transverse momenta tend to zero for high primary energies.

- An apparent increase with primary energy of the temperature $T_{0}$ needed to fit the experimental distributions of transverse momenta between 1 and $10^{6} \mathrm{GeV}$ is qualitatively explained to be a kinematical effect due to a chain of decays leading from the fireballs of the 'first generation' to the observed pions and others. 
- Taking the masses into account, the Boltzmann law becomes $\mathrm{e}^{-\sqrt{p_{\perp}^{2}+m^{2}} / T_{0}}$. For elastic scattering at high energies and outside the diffraction region, this fits the experimental differential cross-section well. If one stresses the formula, one gets into the diffraction region, where the form $\mathrm{e}^{-p_{\perp}^{2} / 2 m T_{0}}$ is correct, but where $T_{0} \approx m_{\pi}$ should be replaced by $\approx 0.4 T_{0}$. If one accepts that, the fit of the diffraction peak is also good. This lower temperature is not inconsistent with the present theory.

These are the results of our paper.

Let us now add some speculations (= wishful thinking). It is possible that the most interesting consequences of our model lie not in explaining so simply the well known but so far completely obscure characteristics of high-energy interactions above $1 \mathrm{GeV}$ - it is possible that the most interesting consequences are to be found in astrophysics and in elementary particle physics. For astrophysics, it is rather obvious: whenever under the influence of gravitational pressure, strong interactions and kinetic energies per particle of the order of $m_{\pi}$ come into play in the centre of a star, the appropriate statistics for a thermodynamical treatment is not Fermi statistics, but the statistics of distinguishable particles. The picture of a 'neutron star' would be inadequate. No work on this question has been done, however. (I thank Dr. G. Cocconi for drawing my attention to this point.)

For elementary particle physics, the following possibility arises. We have seen that the Boltzmann distribution $\mathrm{e}^{-\sqrt{p_{\perp}^{2}+m^{2}} / T}$ fits even the diffraction peak if we have $T \approx 0.4 m_{\pi}$. Since this holds down to $p_{\perp} \rightarrow 0$, where no energy is transferred to the transverse motion and where the collision constitutes a very bad 'position measurement', we feel tempted to conjecture that the incoming particles might already have an 'a priori temperature' (of the order of $0.4 m_{\pi}$ for protons) $T \lesssim T_{0}$ and only a sufficient energy transfer would raise the temperature (for the transverse motion in the c.m. of the collisions) to $T_{0}$. Since the differential elastic cross-section and the total cross-section are related by the optical theorem and since all total cross-sections of hadrons are of the same order at high energies, we would conclude that they all have an 'a priori temperature' of the same order. Then the pion, kaon, nucleon, and hyperons would be thermodynamical systems with a temperature $\approx m_{\pi} / 2$. The immediate question is why, if they are hot, they do not radiate. Why are they all stable against strong decay and the proton even against electromagnetic and weak decay? There are the conservation laws, of course, but they merely state the known facts. They do not explain them. That the proton is stable is not the result of any theory. It has been built in as a postulate in all theories from the beginning. Considering the family of all states with nucleon number 1 and charge 1 , the proton is the ground state of that family not because the results of field theory teach us so, but because we put it in by defining the operators of asymptotic fields that way. Therefore, whether the nucleon is hot or cold, we do not understand why it is stable. Let us therefore ignore this difficulty, which is not typical to the picture which we wish to try. 
We would then have a sequence of different states $\pi, K$, pion resonances, $K$ resonances, $\mathrm{N}$, nucleon resonances, hyperons and their resonances, and so on, leading with ever increasing mass into a continuum called 'fireballs'. All of these systems are more or less well described by thermodynamics of distinguishable particles with temperatures between roughly $0.5 m_{\pi}$ and $m_{\pi}$. For the lowest temperatures, we obtain the stable particles ('strongly' stable) and some new unknown principle is necessary to explain why these states are stable. The missing new principle is analogous to Bohr's quantum condition, which 'explained' why the electrodynamical system called the hydrogen atom had a stable ground state in which the rapidly circulating electron did not radiate-in contradiction to all the then known laws. What we need would be a 'quantum condition' which 'explains' similarly why the thermodynamical system called the proton does not-in contradiction to all the now known laws of the present quantum mechanicsradiated off the rapidly moving particles of which it consists. Maybe the statement that the proton is stable is already the proper form of that postulate, but maybe it could be stated in a more illuminating form as deep as Bohr's $\oint p_{\varphi} \mathrm{d} \varphi=n h$. In any case we cannot be content with that postulate. Bohr's quantum condition for the stability of the proton may later be explained by some generalization of quantum mechanics. It is clear that this condition should give us the mass spectrum of the hadrons, whether the condition itself can be derived from present quantum theory (which is not likely) or from a future generalization of it. It is rather puzzling that not only the spectrum of masses is known to us, but even what corresponds to Wigner's classification of states by group theory, namely, the symmetry schemes $\mathrm{SU}_{3}$, etc., of strong interactions - and in this respect we are far beyond Bohr's $\oint p_{\varphi} \mathrm{d} \varphi=n h$ -but the quantum condition proper is still unknown.

The proton, if our picture should turn out to be true, would then seem to be a straightforward extrapolation of ideas which have been familiar for quite some time. In the early days of renormalization theory, Welton [18] proposed a very intuitive picture of how the vacuum fluctuations shuttle the electron and lead to the observable Lamb shift. It was there considered to be essentially the result of statistical vacuum fluctuations. The interaction, however, is not strong and the $\gamma$ quanta have no mass - the same result could be calculated more exactly with the first few orders of perturbation theory.

In the case of strong interactions, ordinary perturbation theory would not suffice. The large manifold of resonances and fireballs and the short range cause the proton (and all other hadrons) to behave like a thermodynamical system of a rather high (almost the maximal possible) temperature. The 'bootstrap' mechanism would then correspond to a new type of perturbation treatment, in which only the few lowest masses of the unlimited number of interaction 'fireballs' constituting the hadrons are taken into account. It would be a 'first approximation to thermodynamics'.

It is, of course, possible that the picture drawn here is wrong and that the circumstance that our model works even in the diffraction region is purely accidental. But if it is not wrong, then it would follow that we have basically all the information we can hope for in our hands: the mass spectrum, the selection rules $\left(\mathrm{SU}_{3}\right.$, etc.), the decay modes of the lower unstable states $(\rho, \omega$, etc. $)$, and that going to higher and 
higher collisions energies would be comparable to attempting to learn something about the structure of the hydrogen atom by studying the properties of a highly ionized $\mathrm{H}$ gas at higher and higher temperatures.

This latter remark has a good chance to hold even if the above picture of 'a priori hot' hadrons is wrong. The good agreement of our results with experiments indicates that, at least in all collisions with a momentum transfer above $\approx m_{\pi}$, the colliding particles are heated up to the maximal temperature $T_{0} \approx m_{\pi}$. We have therefore little chance of learning much more about the structure of hadrons and about the details of their interaction than we could learn about the structure and interaction of atoms from high-temperature thermodynamics (ideal gases). On the contrary, when we have learnt such things from thermodynamics, it was at low temperatures (condensation, frozen degrees of freedom, superconductivity). If we draw a parallel, we would think that our present laboratory energies below $100 \mathrm{GeV}$ would be the most interesting ones for strong interactions. (We stress once more that not a single one of our conclusions applies to weak and electromagnetic interactions.) One extremely interesting question, however, remains to be answered by high-energy experiments, namely, whether basic triplets for $\mathrm{SU}_{3}$ exist. None of our arguments excludes their being found, e.g., in a $p p$ collision with several $100 \mathrm{GeV}$ in the centre of mass.

Acknowledgements The author is grateful for discussions with A. Bialas, G. Cocconi, B. Escoubès, J. Orear, L. Van Hove and several members of the CERN Theory Division. Mr W. Klein did the numerical calculations for Figs. 19.1 and 19.3.

\section{Appendix 1}

In the main text [Eqs. (19.18) and (19.19)], it was shown that the relative fluctuations in the number of 'fireballs' tend to one:

$$
\frac{\overline{N^{2}}-\bar{N}^{2}}{\bar{N}^{2}} \longrightarrow 1 \text { for } T \rightarrow T_{0} \text {. }
$$

If one defines

$$
w_{\alpha}=\frac{n_{\alpha}}{N},
$$

then $\bar{w}_{\alpha}$ is the probability that a given particle has energy $\varepsilon_{\alpha}$. We wish to show here that

$$
\frac{\overline{w_{\alpha}^{2}}-\bar{w}_{\alpha}^{2}}{\bar{w}_{\alpha}^{2}} \longrightarrow 1 \text { for } T \rightarrow T_{0}
$$


First we note without proof (it is simple) that, if we considered the fluctuations in $\bar{n}_{\alpha}$, they would tend to one. This is not due to the fluctuations with respect to the Boltzmann law, but to the large fluctuations in their normalizing factor $N$. Therefore, we have to consider the fluctuations in $w_{\alpha}$. It is these latter fluctuations which indicate how much we should expect experimental points to scatter about the Boltzmann distribution of transverse momenta.

We write [see Eq. (19.9)]

$$
z=\sum x_{\alpha}, \quad Z=\sum_{N}\left\{\sum_{\sum n=N} \frac{N !}{n_{1} ! \ldots} \prod_{\alpha} x_{\alpha}^{n_{\alpha}}\right\} \equiv \sum_{N} z^{N} .
$$

In order to get $n_{\beta}$ in front of $\prod_{\alpha} x^{n_{\alpha}}$, we should multiply by $x_{\beta} \partial / \partial x_{\beta}$. This operator can be written outside $Z$. Thus $\overline{(n \beta / N)}$ is given by

$$
\overline{\left(\frac{n_{\beta}}{N}\right)}=\frac{1}{Z} x_{\beta} \frac{\partial}{\partial x_{\beta}} \sum_{n} \frac{z^{N}}{N}=\frac{x_{\beta}}{z},
$$

where $\partial / \partial x_{\beta}=\partial / \partial z$ has been used. Similarly,

$$
\overline{\left(\frac{n_{\beta}}{N}\right)^{2}}=\frac{1}{Z}\left(x_{\beta} \frac{\partial}{\partial x_{\beta}}\right)\left(x_{\beta} \frac{\partial}{\partial x_{\beta}}\right) \sum_{n} \frac{z^{N}}{N^{2}}=\frac{1}{Z} x_{\beta}\left(\sum_{N} \frac{z^{N-1}}{N}+x_{\beta} \sum_{N} \frac{N-1}{N} z^{N-2}\right) .
$$

Here the first term in the round bracket is $(\log Z) / z$ and the second tends to $x_{\beta} Z / z^{2}$. For $T \rightarrow T_{0}$, we can neglect $(\log Z) / Z$ to obtain

$$
\overline{\left(\frac{n_{\beta}}{N}\right)^{2}} \longrightarrow\left(\frac{x_{\beta}}{z}\right)^{2}
$$

This, together with Eq. (19.77), proves Eq. (19.75).

A glance at Figs. 19.3 and 19.4 shows that our result agrees with experiment: large fluctuations about the mean multiplicities and small ones about the Boltzmann distribution of transverse momenta.

\section{Appendix 2}

We wish to consider here the broadening of the spectrum due to the decay of a fireball. The problem is straightforward as far as kinematics is concerned. Its general treatment offers, however, great computational difficulties. We therefore treat a simple one-dimensional model case. 
We suppose a fireball with mass $m^{*} \gg T_{0}$ moving along the $x$ axis with a (positive or negative) 'four-velocity' ${ }^{12} V=(\gamma, \beta \gamma)$. This fireball can emit another particle with mass $m_{\ell}$, again only in the $\pm x$ direction, with a 'four-momentum' which is described by $P_{\ell}=\left(\varepsilon_{\ell}, p_{\ell}\right)$ in the fireball's rest frame $F^{*}$, by $P=(\varepsilon, p)$ in the c.m. frame.

We suppose that the momentum distributions of $m^{*}$ in the c.m. frame and the momentum distribution of $m_{\ell}$ in $F^{*}$ are of our standard form for one-dimensional motion [masses fully taken into account, see Eq. (19.51)]:

$$
\begin{array}{ll}
w\left(p^{*}\right) \mathrm{d} p^{*}=\mathrm{e}^{-\varepsilon^{*} / T_{0}} \mathrm{~d} p^{*} & \text { (in c.m. frame) }, \\
w\left(p_{\ell}\right) \mathrm{d} p_{\ell}=\mathrm{e}^{-\varepsilon_{\ell} / T_{0}} \mathrm{~d} p_{\ell} & \left(\text { in } F^{*} \text { frame }\right) .
\end{array}
$$

The four-velocity of the mass $m^{*}$ is given by

$$
V=\left(u_{0}, u\right)=(\gamma, \beta \gamma)=\left(\varepsilon^{*} / m^{*}, p^{*} / m^{*}\right), \quad u_{0}=\sqrt{u^{2}+1}
$$

To the momentum distribution of $m^{*}$ corresponds a velocity distribution

$$
\bar{v}(u)=w\left(p^{*}\right) \frac{\mathrm{d} p^{*}}{\mathrm{~d} m}=m^{*} \mathrm{e}^{-m^{*} \sqrt{u^{2}+1} / T_{0}} .
$$

We normalize this distribution to $\int_{0}^{\infty} v(u) \mathrm{d} u=1$ and obtain

$$
v(u)=\frac{1}{K_{1}\left(m^{*} / T_{0}\right)} \mathrm{e}^{-m^{*} \sqrt{u^{2}+1} / T_{0}},
$$

where $K_{1}$ is as before a Bessel (modified Hankel) function. For $m^{*} \gg T$, we have

$$
K_{1}\left(m^{*} / T_{0}\right) \longrightarrow \sqrt{\frac{2 T_{0}}{\pi m^{*}}} \mathrm{e}^{-m^{*} / T_{0}}
$$

Hence $v(u)$ behaves for small $u$ like $\mathrm{e}^{-m^{*} u^{2} / 2 T_{0}}$ and for large $u$ like $\mathrm{e}^{-m^{*} u / T_{0}}$. Since $m^{*} \gg T_{0}$ was supposed, $v(u)$ drops very fast. Its maximum at $u=0$ increases roughly as $\left(m^{*} / T\right)^{1 / 2}$.

Now let $u$ be fixed for a moment. Then the number of $m_{\ell}$ particles with positive momentum $\{p, \mathrm{~d} p\}$ in the c.m. frame is given by

$$
W_{u}(p) \mathrm{d} p=w\left(p_{\ell}\right) \mathrm{d} p_{\ell},
$$

where $p_{\ell}$ has to be chosen accordingly:

$$
p_{\ell}=p \sqrt{u^{2}+1}-\varepsilon u, \quad \varepsilon_{\ell}=\varepsilon \sqrt{u^{2}+1}-p u .
$$

\footnotetext{
${ }^{12}$ Strictly speaking, we should say 'two-velocity'.
} 
Hence, with $\mathrm{d} p / \mathrm{d} p_{\ell}=\varepsilon_{\ell} / \varepsilon$,

$$
\varepsilon W_{u}(p)=\varepsilon_{\ell}(u) \mathrm{e}^{-\varepsilon_{\ell}(u) / T_{0}} .
$$

This has now to be multiplied by $v(u) \mathrm{d} u$ and integrated from $-\infty$ to $\infty$ (the negative $u$ values take care of the cases in which the fireball moves in the $-x$ direction and emits a 'meson' in the $+x$ direction with sufficient energy to overcompensate the velocity in the $-x$ direction and to obtain a positive direction of flight). Disregarding the normalization factors, we obtain

$$
\varepsilon W(p)=\int_{-\infty}^{\infty} \mathrm{d} u\left[\mathrm{e}^{-m^{*} \sqrt{u^{2}+1} / T_{0}} \mathrm{e}^{-\left(\varepsilon \sqrt{u^{2}+1}-u p\right) / T_{0}}\left(\varepsilon \sqrt{u^{2}+1}-u p\right)\right] .
$$

We know that the first exponential drops first like a Gaussian, later simply exponentially. The exponent in the second exponential is $\varepsilon_{\ell}(u)$ with the following behaviour: $:^{13}$

$$
\varepsilon_{\ell}(u) \longrightarrow \begin{cases}|u|(\varepsilon+p) & \text { for } u \rightarrow-\infty \\ \varepsilon-u p & \text { for } u \approx 0 \\ m_{\ell}(\text { minimal }) & \text { for } u=p / m_{\ell} \\ u(\varepsilon-p) & \text { for } u \rightarrow+\infty\end{cases}
$$

Therefore $\exp \left[-\varepsilon_{\ell}(u) / T\right]$ has a maximum at $u=p / m_{\ell}$ and drops exponentially on both sides. We shall assume that $m^{*}$ is large enough to ensure that the term $\exp \left(-m^{*} \sqrt{u^{2}+1} / T\right)$ can be considered to vary more rapidly than $\exp \left[-\varepsilon_{\ell}(u) / T\right]$, whence the main contributions to the integral will come from $u \approx 0$. Then in the algebraic factor $\varepsilon_{\ell}(u)$ which multiplies the exponentials, we put $u=0$. We expand the square roots in the exponentials and integrate these expressions since the resulting error for large $|u|$ is negligible. Thus omitting all constant factors,

$$
\varepsilon W(p) \approx \varepsilon \mathrm{e}^{-\varepsilon / T_{0}} \int_{-\infty}^{\infty} \exp \left(-u^{2} \frac{\varepsilon+m^{*}}{2 T_{0}}+u \frac{p}{T_{0}}\right) \mathrm{d} u
$$

and

$$
W(p) \approx \sqrt{\frac{2 \pi T_{0}}{\varepsilon+m^{*}}} \mathrm{e}^{-\varepsilon / T_{0}} \mathrm{e}^{p^{2} / 2 T_{0}\left(\varepsilon+m^{*}\right)},
$$

or again,

$$
W(p) \approx \frac{\text { const. }}{\sqrt{\varepsilon+m^{*}}} \exp \left[-\frac{\varepsilon}{T_{0}} \frac{2\left(\varepsilon+m^{*}\right)-p^{2} / \varepsilon}{2\left(\varepsilon+m^{*}\right)}\right]
$$

\footnotetext{
${ }^{13} \varepsilon_{\ell}(u)$ has the maximum possible value $m^{*} / 2$, namely, when $m^{*} \rightarrow m_{\ell}+m_{\ell}$. In general, $\varepsilon_{\ell}$ is much smaller. In the integral, we forget about this fact, since large $u$ values contribute nothing.
} 
We may then write

$$
W(p) \approx \frac{\text { const. }}{\sqrt{\varepsilon+m^{*}}} \mathrm{e}^{-\varepsilon / T_{\text {eff }}}
$$

where

$$
T_{\mathrm{eff}}(\varepsilon)=T_{0} \frac{2\left(\varepsilon+m^{*}\right)}{2\left(\varepsilon+m^{*}\right)-p^{2} / \varepsilon}, \quad p^{2}=\varepsilon^{2}-m_{\ell}^{2} .
$$

When $\varepsilon$ varies between $m_{\ell}$ and $\infty, T_{\text {eff }}(\varepsilon)$ varies between $T_{0}$ and $2 T_{0}$, the latter value is approached only for $\varepsilon$ values $\gg m^{*}$.

In cosmic ray jets, transverse momenta of pions up to the order $p=\varepsilon=1$ to 1.2 have been reliably measured [11]. Assume then $p=\varepsilon=1$ and $m^{*}=1$. Then $T_{\text {eff }}=4 T_{0} / 3$ and with $m^{*}=2$, we find $T_{\text {eff }}=6 T_{0} / 5$. This is at the upper end of the measured spectrum. At the lower end, $T_{\text {eff }} \rightarrow T_{0}$.

The present analysis is very rough and incomplete. It illustrates only the mechanism. It is not inconsistent with the assumption that nothing serious would happen in reality (the two-dimensional case). If that turned out to be so and if the general case gave a similar result, then it would allow one to conclude from the transverse momentum distribution [namely, the deviations from a pure $\exp \left(-\varepsilon / T_{0}\right)$ ] something about the average or most frequent mass $m^{*}$ of fireballs. The heavier the fireballs, the less the actual distribution will deviate from an exponential. On the other hand, the chain of decays will then contain more members and this may increase the deviations again. In any case it will at least cause a larger effective temperature. Actually, the $T$ which is needed to fit the spectra seems to increase somewhat with the primary energy, although not more than by a factor of two, when the primary energy varies by a factor of one million.

In spite of this very crude analysis, we believe that it is sufficient to make it very likely that the apparent increase in the temperature is entirely due to kinematics and that our $T_{0}$ is indeed independent of the primary energy.

A more careful and more realistic (two-dimensional) discussion of this problem is highly desirable.

Open Access This book is distributed under the terms of the Creative Commons Attribution Noncommercial License which permits any noncommercial use, distribution, and reproduction in any medium, provided the original author(s) and source and credited.

\section{References}

1. E. Fermi, Prog. Theor. Phys. 5, 570 (1950)

2. G. Fast, R. Hagedorn, Nuovo Cimento 27, 208 (1963)

3. G. Fast, R. Hagedorn, L.W. Jones, Nuovo Cimento 27, 856 (1963)

4. R. Hagedorn, Nuovo Cimento 35, 216 (1965) 
5. G. Auberson, B. Escoubés, Nuovo Cimento 36, 628 (1965)

6. A. Bialas, V.F. Weisskopf, Nuovo Cimento 35, 1211 (1965)

7. J. Vandermeulen, Bull. Soc. R. Sci. Liège (Belgium) 34, 455 (1965)

8. H. Satz, The High Energy Limit of the Statistical Model, August 1964. Summer Institute for Theoretical Physics, University of Wisconsin, Madison, Wisc., USA; now at DESY, Hamburg, Germany

9. G. Cocconi, Nuovo Cimento 33, 643 (1964). Cocconi says: "[...] corresponds to the case of a system in which, for $E$ increasing, the number of possible kinds of particles increases so as to keep the energy per particle, and hence the temperature, constant." In this remark, our whole theory is implicitly contained

10. T.T. Wu, C.N. Yang, Phys. Rev. 137, B708 (1965)

11. P.H. Fowler, D.H. Perkins, Proc. R. Soc. A 278, 401 (1964)

12. D. Amati, A. Stanghellini, S. Fubini, Nuovo Cimento 26, 896 (1962)

13. K.A. Wilson, Acta Phys. Anstriaca 17, 37 (1963)

14. J. Orear, Phys. Rev. Lett. 12, 112 (1964); Phys. Lett. 13, 190 (1965)

15. G. Cocconi, L.J. Koester, D.H. Perkins, Lawrence Radiation Laboratory Rep. UCID-1444 (1961)

16. M.L. Goldberger, K.M. Watson, Collision Theory (Wiley, New York, 1964)

17. T.D. Newton, E.P. Wigner, Rev. Mod. Phys. 21, 400 (1949)

18. T. Welton, Phys. Rev. 74, 1157 (1948) 